INSTITUT NATIONAL DE RECHERCHE EN INFORMATIQUE ET EN AUTOMATIQUE

\title{
Spectral Theorem for Convex Monotone Homogeneous Maps, and Ergodic Control
}

\author{
Marianne Akian — Stéphane Gaubert
}

$\mathbf{N}^{\circ} \mathbf{4 2 7 3}$

Octobre 2001

THÈME 4 



\title{
Spectral Theorem for Convex Monotone Homogeneous Maps, and Ergodic Control
}

\author{
Marianne Akian*, Stéphane Gaubert ${ }^{\dagger}$ \\ Thème 4 - Simulation et optimisation \\ de systèmes complexes \\ Projet Metalau \\ Rapport de recherche $n^{\circ} 4273$ - Octobre 2001 - 44 pages
}

\begin{abstract}
We consider convex maps $f: \mathbb{R}^{n} \rightarrow \mathbb{R}^{n}$ that are monotone (i.e., that preserve the product ordering of $\mathbb{R}^{n}$ ), and nonexpansive for the sup-norm. This includes convex monotone maps that are additively homogeneous (i.e., that commute with the addition of constants). We show that the fixed point set of $f$, when it is non-empty, is isomorphic to a convex inf-subsemilattice of $\mathbb{R}^{n}$, whose dimension is at most equal to the number of strongly connected components of a critical graph defined from the tangent affine maps of $f$. This yields in particular an uniqueness result for the bias vector of ergodic control problems. This generalizes results obtained previously by Lanery, Romanovsky, and Schweitzer and Federgruen, for ergodic control problems with finite state and action spaces, which correspond to the special case of piecewise affine maps $f$. We also show that the length of periodic orbits of $f$ is bounded by the cyclicity of its critical graph, which implies that the possible orbit lengths of $f$ are exactly the orders of elements of the symmetric group on $n$ letters.

Key-words: Nonexpansive maps, Periodic orbits, Eigenspace, Spectral theorem, Stochastic Control, Ergodic Control, Perron-Frobenius Theorem, Max-plus algebra, Critical graph, Convexity, Subdifferentials
\end{abstract}

This work was partially supported by the European Community Framework IV program through the research network ALAPEDES ("The Algebraic Approach to Performance Evaluation of Discrete Event Systems"). The results of this paper were announced in [AG01].

* Email: Marianne.Akian@inria.fr

$\dagger$ ENSTA, 32 Bd. Victor, 78539 Paris Cédex 15, France, and INRIA Rocquencourt. Email: Stephane.Gauberteinria.fr 


\section{Théorème spectral pour les fonctions monotones homogènes convexes, et Contrôle ergodique}

Résumé : Nous considérons les applications $f: \mathbb{R}^{n} \rightarrow \mathbb{R}^{n}$ qui sont convexes, monotones (i.e. qui preservent l'ordre produit de $\mathbb{R}^{n}$ ), et contractantes au sens large pour la norme du sup. Cela comprend les applications monotones, convexes qui sont additivement homogènes (i.e., qui commutent avec l'addition des constantes). Nous montrons que l'ensemble des points fixes de $f$, lorsqu'il est non vide, est isomorphe à un inf-sous-semitreillis convexe de $\mathbb{R}^{n}$, dont la dimension est au plus égale au nombre de composantes fortement connexes d'un graphe critique, lui même construit à partir des applications affines tangentes à $f$. On en déduit, en particulier, un résultat d'unicité pour la fonction de biais de problèmes de contrôle ergodique. Notre résultat généralise des résultats plus anciens, obtenus par Lanery, Romanovsky, et Schweitzer et Federgruen, pour les problèmes de contrôle ergodique avec espaces d'états et d'actions finis, ce qui correspond au cas particulier des applications $f$ affines par morceaux. Nous montrons aussi que la longueur des orbites périodiques de $f$ est bornée par la cyclicité de son graphe critique, ce qui implique que les longueurs possibles des orbites de $f$ sont exactement les ordres des permutations à $n$ éléments.

Mots-clés : Applications contractantes, Orbites périodiques, Éspace propre, Théorème spectral, Contrôle stochastique, Contrôle ergodique, Théorème de Perron-Frobenius, Algèbre max-plus, Graphe critique, Convexité, Sous-différentiels 


\section{Contents}

1 Introduction $\quad 3$

1.1 Motivations and statement of the main result . . . . . . . . . . . . . . . 3

1.2 Related optimal control results . . . . . . . . . . . . . . . . . . 6

1.3 From Uniqueness Results to Existence Results . . . . . . . . . . . . . . . . . . . . . 7

1.4 Extension to convex monotone subhomogeneous maps . . . . . . . . . . . 8

2 Class Structure of Convex Monotone Homogeneous Maps 9

2.1 Subdifferentials of Convex Monotone Homogeneous Maps . . . . . . . . . . . . . . 9

2.2 Convex Rectangular Sets of Stochastic Matrices . . . . . . . . . . . . . . . . . . . 10

2.3 Critical Graph of Convex Monotone Homogeneous Maps . . . . . . . . . . . . . . . 12

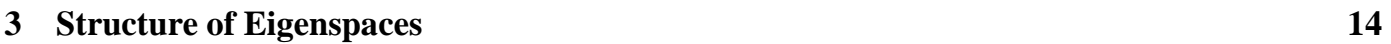

4 Critical Graph of $f^{k} \quad 19$

5 Cyclicity Theorem for Convex Monotone Homogeneous Maps 25

6 Piecewise Affine Convex Monotone Homogeneous Maps 27

6.1 Dimension of the eigenspace . . . . . . . . . . . . . . . . 27

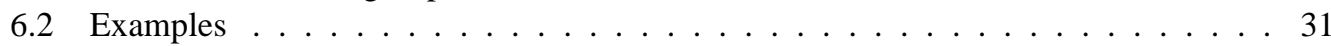

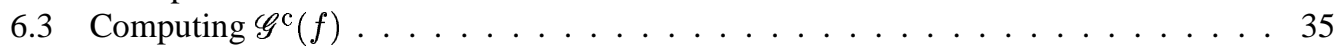

7 Stochastic Control Interpretation 3

\section{Introduction}

\subsection{Motivations and statement of the main result}

We say that a map $f: \mathbb{R}^{n} \rightarrow \mathbb{R}^{n}$ is monotone if for all $x, y \in \mathbb{R}^{n}, x \leq y \Longrightarrow f(x) \leq f(y)$, where $\leq$ denotes the product ordering of $\mathbb{R}^{n}\left(x \leq y\right.$ if $x_{i} \leq y_{i}$, for all $\left.1 \leq i \leq n\right)$. We say that $f$ is additively homogeneous if for all $\lambda \in \mathbb{R}, x \in \mathbb{R}^{n}, f(\lambda+x)=\lambda+f(x)$, where $\lambda+x=$ $\left(\lambda+x_{1}, \ldots, \lambda+x_{n}\right)$. It is easy to see that a monotone homogeneous map is nonexpansive for the sup-norm: for all $x, y \in \mathbb{R}^{n},|f(x)-f(y)| \leq|x-y|$, where $|x|=\max _{1 \leq i \leq n}\left|x_{i}\right|$ (see [CT80]).

Monotone homogeneous maps arise classically in optimal control and game theory (see for instance [Whi86, Kol92, FV97, RS01]), in the modeling of discrete events systems (see [BCOQ92, Gun94, CGQ95a, CGQ95b, Vin97, GG98, Gun01]), and in nonlinear potential theory [De196], as nonlinear extension of Markov transitions. They also arise in nonlinear PerronFrobenius theory, when one considers multiplicatively homogeneous maps $F$ acting on a cone and preserving the order of the cone: in the simplest case, when the cone is $\left(\mathbb{R}_{+}^{*}\right)^{n}$ (where $\mathbb{R}_{+}^{*}=\{x \in \mathbb{R} \mid x>0\}$ ), the transformation $F \mapsto \log \circ F \circ \exp \left(\right.$ where log $:\left(\mathbb{R}_{+}^{*}\right)^{n} \rightarrow \mathbb{R}^{n}$ 
is the map which does $\log$ entrywise, and $\exp =\log ^{-1}$ ) sends the set of monotone multiplicatively homogeneous maps to the set of monotone additively homogeneous maps. See for instance [Bou95, LW94, Mor64, MS69, Nus88, Nus89, Sab97, Woj85] for various studies and applications.

A basic problem, for a monotone homogeneous map $f$, is the existence, and uniqueness (up to an additive constant), of the additive eigenvectors of $f$, which are the $v \in \mathbb{R}^{n}$ such that $f(v)=\lambda+v$, for some additive eigenvalue $\lambda \in \mathbb{R}$. In the sequel, we will omit the term "additive", when the additive nature of the objects will be clear from the context. When $f$ has an eigenvector $v$ with eigenvalue $\lambda$, by homogeneity of $f, f^{k}(v)=k \lambda+v$ holds for all $k \geq 0$, and by nonexpansiveness of $f,\left|f^{k}(x)-k \lambda-v\right|=\left|f^{k}(x)-f^{k}(v)\right| \leq|x-v|$, hence,

$$
f^{k}(x)=k \lambda+O(1) \quad \text { when } k \rightarrow \infty,
$$

for all $x \in \mathbb{R}^{n}$ (all the orbits of $f$ have a linear growth rate of $\lambda$ ). This implies in particular that the eigenvalue $\lambda$ is unique. Hence, we can speak without ambiguity of the eigenspace of $f$, which is the set $\mathscr{E}(f)=\left\{x \in \mathbb{R}^{n} \mid f(x)=\lambda+x\right\}$. In many applications, the eigenvalue and eigenvector are fundamental objects: for instance, in stochastic control, the eigenvalue gives the optimal reward per time unit, and eigenvectors give stationary rewards (we explain this in detail in Section 7). In discrete event systems applications, the eigenvalue gives the throughput, and eigenvectors give stationary schedules.

Several Perron-Frobenius like theorems, guarantee the existence of eigenvectors of monotone (additively) homogeneous maps $\mathbb{R}^{n} \rightarrow \mathbb{R}^{n}$. Such results go back at least to Krĕ̌n and Rutman [KR50, §7], in the context of monotone multiplicatively homogeneous maps leaving a cone in a Banach space invariant, and to Morishima, whose book [Mor64] contains a complete study of finite dimensional non-linear Perron-Frobenius theory. A modern overview appears in the memoirs of Nussbaum [Nus88, Nus89], which contain in particular general existence results for eigenvectors, following [Nus86]. Different existence conditions appeared in [Osh84]. General results on the geometry of the eigenspaces are available, for instance, the result of Bruck [Bru73] shows in particular that $\mathscr{E}(f)$ is the image of a nonexpansive projector and a fortiori is connected, see also [Nus88, Theorems 4.5, 4.6 and 4.7].

In this paper, we describe the eigenspaces of convex monotone homogeneous maps $f: \mathbb{R}^{n} \rightarrow$ $\mathbb{R}^{n}$. (We say that a $\mathbb{R}^{n}$-valued map is convex when its coordinates are convex. We refer the reader to [Roc70] for all convexity notions used in the paper: subdifferentials, domain, Fenchel transform, etc.)

To state our main result, we need a few definitions (see Section 2 for details). We first generalize the notion of subdifferential to maps $\mathbb{R}^{n} \rightarrow \mathbb{R}^{n}$ by setting, for $x \in \mathbb{R}^{n}, \partial f(x)=\left\{P \in \mathbb{R}^{n \times n} \mid\right.$ $\left.f(y)-f(x) \geq P(y-x), \forall y \in \mathbb{R}^{n}\right\}$. It is easy to see (Corollary 2.2 and Equation (4) below) that by monotonicity and homogeneity of $f$, the elements of $\partial f(x)$ are stochastic matrices. If $v$ is an eigenvector of $f$, we call critical graph of $f$, the (directed) graph $\mathscr{G}^{\mathrm{c}}(f)$ which is the union of final graphs of stochastic matrices $P \in \partial f(v)$ (we call final graph of a stochastic matrix the restriction of its graph to the set of final classes, see $\$ 2.2$ and $\$ 2.3)$. The graph $\mathscr{G}^{\mathrm{c}}(f)$ is independent of the choice of the eigenvector $v$ (Proposition 2.5 below). We call critical nodes of $f$, the nodes of $\mathscr{G}^{\mathrm{c}}(f)$, and denote by $\mathrm{N}^{\mathrm{c}}(f)$ the set of critical nodes. We call critical classes of $f$ the sets of nodes 
$C_{1}, \ldots, C_{s}$ of the strongly connected components of $\mathscr{G}^{\mathrm{c}}(f), \mathscr{G}_{1}, \ldots, \mathscr{G}_{s}$. We call cyclicity of $\mathscr{G}_{i}$, and denote by c $\left(\mathscr{G}_{i}\right)$, the gcd of the lengths of the circuits of $\mathscr{G}_{i}$, and we define the cyclicity of $f$ by $\mathrm{c}(f)=\operatorname{lcm}\left(\mathrm{c}\left(\mathscr{G}_{1}\right), \ldots, \mathrm{c}\left(\mathscr{G}_{s}\right)\right)$. We say that a monotone homogeneous map $g: U \subset \mathbb{R}^{n} \rightarrow V \subset \mathbb{R}^{p}$ is a monotone homogeneous isomorphism if it has a monotone homogeneous inverse. The following theorem gathers results from Theorem 3.4, Corollary 3.6, Corollary 5.7, and Theorem 6.6 below.

Theorem 1.1 (Convex Spectral Theorem). Let $f: \mathbb{R}^{n} \rightarrow \mathbb{R}^{n}$ denote a convex monotone homogeneous map that has an eigenvector. Denote by $C=\mathrm{N}^{\mathrm{c}}(f)$ the set of critical nodes of $f, c=\mathrm{c}(f)$ the cyclicity of $f$, and $\lambda$ the unique eigenvalue of $f$. Then,

1. the restriction $\mathrm{r}: \mathbb{R}^{n} \rightarrow \mathbb{R}^{C}, x \mapsto\left(x_{i}\right)_{i \in C}$, is a monotone homogeneous isomorphism from $\mathscr{E}(f)$ to its image $\mathscr{E}^{\mathrm{c}}(f)$;

2. $\mathscr{E}^{\mathrm{c}}(f)$ is an inf-subsemilattice of $\left(\mathbb{R}^{C}, \leq\right)$;

3. $\mathscr{E}^{\mathrm{c}}(f)$ is a convex set whose dimension is at most equal to the number of critical classes of $f$, and this bound is attained when $f$ is piecewise affine;

4. for all $x \in \mathbb{R}^{n}, f^{k c}(x)-k c \lambda$ has a limit when $k \rightarrow \infty$.

In particular, when $f$ has only one critical class, the eigenvector of $f$ is unique (up to an additive constant). It also follows from the last assertion of the theorem that the set of limit points of $f^{k}(x)-$ $k \lambda$ when $k \rightarrow \infty$, and $x \in \mathbb{R}^{n}$, is precisely $\mathscr{E}\left(f^{c}\right)$. Theorem 1.1 also allows us to bound the dimension of this set. Indeed, we shall see in Theorem 4.1 and Proposition 5.3 below that the set of critical nodes is the same for $f$ and $f^{c}$, and that $f^{c}$ has $\mathrm{c}\left(\mathscr{G}_{1}\right)+\cdots+\mathrm{c}\left(\mathscr{G}_{s}\right)$ critical classes. Hence, applying Theorem 1.1 to $f^{c}$, we get that the restriction $r$ is a monotone homogeneous isomorphism from $\mathscr{E}\left(f^{c}\right)$ to a convex set, $\mathscr{E}^{c}\left(f^{c}\right)$, of dimension at most $\mathrm{c}\left(\mathscr{G}_{1}\right)+\cdots+\mathrm{c}\left(\mathscr{G}_{s}\right)$, the bound being attained when $f$ is piecewise affine.

The paper is devoted to the proof (Sections 2-6) and to the stochastic control interpretation (Section 7) of Theorem 1.1. In Section 2, we detail the definitions and properties of subdifferentials and critical graph of convex monotone homogeneous maps. An important element of the proofs is the maximum principle for Markov chains (Lemma 2.9). In Section 3, we establish the first part of Theorem 1.1 concerning the structure of the eigenspace: points 1,2 and the first assertion in point 3. The main argument is again the maximum principle. Section 4 is devoted to further tools and properties used in the remaining sections, which are of independent interest: Theorem 4.1 shows that $\mathscr{G}^{\mathrm{c}}\left(f^{k}\right)=\mathscr{G}^{\mathrm{c}}(f)^{k}$ (this will be used in Section 5 for the proof of the cyclicity part of Theorem 1.1; we also introduce directional derivatives (which will be used in Section 6 to connect $\mathscr{E}(f)$ to $\mathscr{E}\left(f_{v}^{\prime}\right)$ for any eigenvector $v$ ), additive recession functions (formula (15)), invariant critical classes and the associated decomposition of $f$ (Lemma 4.9), and also a characterization of the set of critical nodes in terms of supports of nonlinear "excessive" measures (Proposition 4.5).

Section 5 is devoted to the proof of point 4 of Theorem 1.1. This result relies on a more general theorem of Nussbaum [Nus90] and Sine [Sin90], which states that if $f: \mathbb{R}^{n} \rightarrow \mathbb{R}^{n}$ is nonexpansive for the sup-norm and has a fixed point, then, for all $x \in \mathbb{R}^{n}, f^{k c}(x)$ converges when $k \rightarrow \infty$, for some minimal constant $c$ which can be bounded by a function of $n$. When $f$ is convex monotone and homogeneous, the last assertion of the convex spectral theorem shows that the possible values of $c$ are 
exactly the orders of elements of the symmetric group on $n$ letters. Equivalently, convex monotone homogeneous maps have the same orbit lengths as permutation matrices, a result which was known to be true in the special cases of linear maps associated to nonnegative matrices (see [NVL99, chapter 9]), of linear maps over the max-plus semiring (see [CDQV83, Nus91a]), and also of piecewise affine convex monotone homogeneous maps (which include max-plus linear maps), see the discussion in $\S 1.2$ below. More generally, computing the orbit lengths of nonexpansive maps for polyhedral norms raises interesting combinatorial and analytical problems (see in particular [AK87, Wel87, Sch88, Sch91, Nus90, Nus91b, NSVL98, NVL99, Lem01]).

The equality in point 3 of Theorem 1.1 is proved in Section 6 . As will be discussed in $\S 1.2$, this part of the theorem has already been proved by Romanovsky [Rom73] and by Schweitzer and Federgruen [SF78]. We provide here an independent proof, which emphasizes the qualitative properties of $\mathscr{E}(f)$, using the tools of Section 4. We also give a polynomial time algorithm to compute $\mathscr{G}^{\mathrm{c}}(f)$.

\subsection{Related optimal control results}

Convex monotone homogeneous maps $f: \mathbb{R}^{n} \rightarrow \mathbb{R}^{n}$ are exactly dynamic programming operators associated to stochastic control problems with state space $\{1, \ldots, n\}$. Computing the stationary solutions and the asymptotic behavior of solutions of dynamic programming equations is an old problem of stochastic control which is essentially equivalent to that of computing the eigenspace $\mathscr{E}(f)$ and the asymptotics of $f^{k}$ when $k \rightarrow \infty$. This has been much studied in the stochastic control literature, particularly in the case of finite action spaces, which corresponds to piecewise affine maps. In this special case, results equivalent to the third assertion of Theorem 1.1 were obtained by Romanovsky [Rom73] using linear programming techniques, and also by Schweitzer and Federgruen [SF78] who gave an explicit representation of $\mathscr{E}(f)$ in terms of resolvents associated to optimal strategies (see [SF78, Theorem 4.1]). Again in this special case, a result equivalent to the fourth assertion of Theorem 1.1 was stated by Lanery [Lan67]. The arguments of [Lan67] only proved the special case where $\mathrm{N}^{\mathrm{c}}(f)=\{1, \ldots, n\}$, see the discussion in the introduction and in Note 1 of [SF77]. A proof valid for a general $\mathrm{N}^{c}(f) \subset\{1, \ldots, n\}$ was given by Schweitzer and Federgruen [SF77], who also proved the optimality of $c(f)$.

The special case of deterministic control problems leads to maps $f$ that are max-plus linear. These maps have been studied independently by the max-plus community. In this context, the dimension of the eigenspace was characterized by Gondran and Minoux [GM77], and the remaining part of the max-plus spectral theorem, dealing with cyclicity, was obtained by Cohen, Dubois, Quadrat, and Viot [CDQV83] (see also [BCOQ92]). (Note however that more precise results -explicit form of the eigenspace, finite time convergence of the iterates- are available in the max-plus case.) The max-plus spectral theorem has a long story, which goes back to Cuninghame-Green (see [CG79] and the references therein), Romanovsky [Rom67], and Vorobyev [Vor67], to quote the most ancient contributions. See the collection of articles [MS92], Kolokoltsov and Maslov [KM97], and the references therein, for generalizations to infinite dimension. See also [GP97, Bap98] for surveys.

The present work was inspired by the max-plus spectral theorem and uses nonexpansive maps techniques (we were unaware of the results of [Lan67, Rom73, SF77, SF78]). We next emphasize differences with earlier results. We consider general convex monotone homogeneous maps, 
which correspond to stochastic control problems with finite state space and arbitrary action spaces, whereas the results in [Lan67, Rom73, SF77, SF78] require the action space to be finite. Our proof technique, which relies on the maximum principle, can be naturally transposed to other (infinite dimensional) contexts. Another tool in our proof is the critical graph $\mathscr{G}^{\mathrm{c}}(f)$, which generalizes the critical graph that appears in max-plus algebra (see Proposition 2.7). The critical graph already appeared in [Rom73, p. 491], with a different definition in terms of optimal policies. The new definition that we give here in terms of subdifferentials leads in particular to a polynomial time algorithm to compute $\mathscr{G}^{\mathrm{c}}(f)$ (see $\S 6.3$ ). (The equivalence of both definitions is shown in Proposition 7.2.) It should also be noted that when passing from the case of finite action spaces to arbitrary action spaces, new phenomena occur. For instance, Example 3.9 shows that we cannot hope, in general, to characterize the dimension of eigenspaces in terms of graphs like $\mathscr{G}^{\mathrm{c}}(f)$.

Let us mention in passing that the critical graph has an intuitive interpretation in terms of "recurrence". For a Markov chain, a node is recurrent if the probability of return to this node is equal to one. For a max-plus matrix with eigenvalue 0 , a node is "recurrent", i.e. belong to a critical class, if we can return to this node with zero reward. When $f$ is a convex monotone homogeneous map with eigenvalue 0 , a node $i$ is "recurrent", i.e. belong to a critical class, if we can find a strategy for which, starting from $i$, we eventually return to $i$ with probability 1 and zero mean reward. This provides a new illustration of the analogy between probability and optimization developed in [Mas73, Chap. VIII], [MTRS90, Qua90, AQV95, Mor95], [QP97, §4.2], [LMS00], and [Puh01].

Ergodic control problems of diffusion processes lead to spectral problems for infinite dimensional monotone homogeneous semigroups which can be expressed in terms of ergodic HamiltonJacobi-Bellman (HJB) partial differential equations. In [Ben88], Bensoussan proved uniqueness of the eigenvector (as weak solution of the ergodic HJB equation) under assumptions, which translated in finite dimension imply irreducibility of stochastic matrices $P \in \partial f(v)$. Inspired by the results of the present paper, the first author, Sulem and Taksar [AST01] proved uniqueness of the viscosity solution of a special ergodic HJB equation. This yields an example of concrete situation where some non optimal stationary strategies have several final classes, whereas the optimal ones have only one final class (translated to our setting, this means that for some $x \in \mathbb{R}^{n}$ and $P \in \partial f(x), P$ may have several final classes, whereas there exists an eigenvector $v$ such that all elements of $\partial f(v)$ have one final class).

\subsection{From Uniqueness Results to Existence Results}

The uniqueness result that follows from Theorem 1.1 can be thought of as a partial extension of the condition of Nussbaum [Nus88, Theorem 2.5]: specialized to convex monotone homogeneous maps $f: \mathbb{R}^{n} \rightarrow \mathbb{R}^{n}$, the result of Nussbaum shows that if $f$ is $\mathcal{C}^{1}$ and for all eigenvectors $v, f^{\prime}(v)$ has only one final class (in this case of course $f$ has a unique critical class), then the eigenvector of $f$ is unique. The idea of all these results is that the dimension of $\mathscr{E}(f)$ can be bounded by looking at "linearizations" of $f$ near an eigenvector.

It is instructive to note that the uniqueness of eigenvectors in $\mathbb{R}^{n}$ is governed by the same kind of graph properties as the existence of eigenvectors, albeit the graphs are different. For instance, a result of the second author and Gunawardena [GG01, Theorem 2] guarantees the existence of an 
eigenvector for a monotone homogeneous map which has a strongly connected graph. Here, the $\operatorname{graph} \mathscr{G}(f)$ of a monotone homogeneous map $f: \mathbb{R}^{n} \rightarrow \mathbb{R}^{n}$ has nodes $\{1, \ldots, n\}$ and an arc $i \rightarrow j$ if $\lim _{\nu \rightarrow \infty} f_{i}\left(\nu e_{j}\right)=+\infty$, where $e_{j}$ denotes the $j$-th vector of the canonical basis of $\mathbb{R}^{n}$. Another way to guarantee the existence of an eigenvector is to use the convex spectral theorem itself, thanks to the following observation taken from [GG01]. We denote by $\widehat{f}(x)=\lim _{\mu \rightarrow \infty} \mu^{-1} f(\mu x)$ the recession function of $f$ ( $\widehat{f}$ need not exist when $f$ is monotone and homogeneous, but it does exist when $f$ is convex). We have $\widehat{f}(0)=0$, and when $f$ is (additively) homogeneous, so does $\widehat{f}$, so that all points on the diagonal are (trivial) fixed points of $f$. It is proved in [GG01] that if the recession function of a monotone homogeneous map $f$ exists and has only fixed points on the diagonal, then, $f$ has an eigenvector. Combining this observation with the convex spectral theorem, we obtain:

Corollary 1.2. A monotone homogeneous map has an eigenvector if its recession function exists, is convex, and has only one critical class.

If $f$ is a convex monotone homogeneous map, it is not difficult to see that the recession function $\widehat{f}$ is exactly the support function of the domain of the Fenchel transform $f^{*}$ of $f$ (defined in Section 2.1 below), that is $\widehat{f}(x)=\sup _{P \in \operatorname{dom} f^{*}} P x$. In this formula, one can replace dom $f^{*}$ by its closure $\operatorname{cl}\left(\operatorname{dom} f^{*}\right)$, which is equal to $\partial \widehat{f}(0)$. The graph $\mathscr{G}(f)$ is the union of the graphs of $P \in \operatorname{dom} f^{*}$, or equivalently the union of the graphs of $P \in \operatorname{cl}\left(\operatorname{dom} f^{*}\right)$, whereas the critical graph $\mathscr{G}^{\mathrm{c}}(\widehat{f})$ is the union of the final graphs of $P \in \operatorname{cl}\left(\operatorname{dom} f^{*}\right)$. If $\mathscr{G}(f)$ is strongly connected, one can see that $\mathscr{G}^{\mathrm{C}}(\widehat{f})$ is also strongly connected, so that in the special case of convex monotone homogeneous maps, Corollary 1.2 is stronger than Theorem 2 of [GG01] (which however holds in a more general context).

\subsection{Extension to convex monotone subhomogeneous maps}

Finally, let us mention two immediate extensions of the convex spectral theorem. First, since the map $f \mapsto(x \mapsto-f(-x))$ sends convex monotone homogeneous maps to concave monotone homogeneous maps, there is of course a dual concave spectral theorem. Another, more interesting, extension, is obtained by considering subhomogeneous maps $f$, which satisfy $f(\lambda+x) \leq \lambda+f(x)$, for all $\lambda \geq 0$ and $x \in \mathbb{R}^{n}$. It is easy to see that a monotone map is subhomogeneous if, and only if, it is nonexpansive for the sup-norm. To a monotone subhomogeneous map $f: \mathbb{R}^{n} \rightarrow \mathbb{R}^{n}$, we associate canonically a monotone homogeneous map $g: \mathbb{R}^{n+1} \rightarrow \mathbb{R}^{n+1}$,

$$
g(x, y)=\left(\begin{array}{c}
y+f(-y+x) \\
y
\end{array}\right), \quad \forall x \in \mathbb{R}^{n}, y \in \mathbb{R}
$$

(this is a nonlinear extension of the classical way of passing from a substochastic to a stochastic matrix, by adding a cemetery state, in this non-linear context, this construction is due to Gunawardena and Keane [GK95]). A vector $z \in \mathbb{R}^{n}$ is a fixed point of $f$, if, and only if $(z, 0)$ is an eigenvector of $g$ (and the eigenvalue is 0). Using this construction, one translates readily the Convex Spectral Theorem to a theorem describing fixed point sets and the asymptotics of the iterates of convex monotone subhomogeneous maps. (We might also use this construction, with $-\lambda+f$ instead of $f$, to describe 
the eigenspace of $f$ for an additive eigenvalue $\lambda$, but when $f$ is only monotone and subhomogeneous, $\lambda$ need not be unique, and it tells little about the asymptotics of $f^{k}$, in general.) For a convex monotone subhomogeneous map $f$ with fixed point $v$, the critical graph $\mathscr{G}^{\mathrm{c}}(f)$ of $f$ is defined as the union of the graphs of the matrices $P_{C C}$, where $P \in \partial f(v), C$ is a final class of $P$, and the $C \times C$ submatrix of $P, P_{C C}$, is stochastic (when $f$ is homogeneous, this property is automatically satisfied). Equivalently, $\mathscr{G}^{\mathrm{c}}(f)$ (which can be empty) is the restriction of $\mathscr{G}^{\mathrm{c}}(g)$ to $\{1, \ldots, n\}$. The notions of critical classes and cyclicity are defined from $\mathscr{G}^{\mathrm{c}}(f)$ as above. When $\mathscr{G}^{\mathrm{c}}(f)$ is empty, we have $\mathrm{N}^{\mathrm{c}}(f)=\varnothing$, and we take the convention $\mathbb{R}^{\varnothing}=\{0\}$, and $\mathrm{c}(f)=1$.

Corollary 1.3. Let $f: \mathbb{R}^{n} \rightarrow \mathbb{R}^{n}$ denote a convex monotone subhomogeneous map that has a fixed point. Then, all the conclusions of the Convex Spectral Theorem apply to $f$ and $\lambda=0$. In particular, if $f$ has no critical classes, then its fixed point is unique.

Acknowledgements. The authors thank J. Gunawardena, J.P. Quadrat, and C. Sparrow, for many useful discussions.

\section{Class Structure of Convex Monotone Homogeneous Maps}

\subsection{Subdifferentials of Convex Monotone Homogeneous Maps}

We shall first consider scalar monotone homogeneous maps $g: \mathbb{R}^{n} \rightarrow \mathbb{R}$ (which satisfy $x \leq y \Longrightarrow$ $g(x) \leq g(y)$ for all $x, y \in \mathbb{R}^{n}$, and $g(\lambda+x)=\lambda+g(x)$, for all $\lambda \in \mathbb{R}$ and $\left.x \in \mathbb{R}^{n}\right)$. The Fenchel transform of $g$ is the map $g^{*}: \mathbb{R}^{n} \rightarrow \mathbb{R} \cup\{+\infty\}, g^{*}(p)=\sup _{x \in \mathbb{R}^{n}}(p \cdot x-g(x))$. We denote by $\operatorname{dom} g^{*}=\left\{p \in \mathbb{R}^{n} \mid g^{*}(p)<\infty\right\}$ the domain of $g^{*}$, and by $\mathscr{S}_{n}=\left\{p \in \mathbb{R}^{n} \mid \sum_{1 \leq i \leq n} p_{i}=\right.$ $\left.1, p_{1}, \ldots, p_{n} \geq 0\right\}$ the set of stochastic vectors.

Proposition 2.1. If $g: \mathbb{R}^{n} \rightarrow \mathbb{R}$ is monotone and homogeneous, then, dom $g^{*}$ is included in $\mathscr{S}_{n}$.

Proof. Let $1_{n}$ denote the vector of $\mathbb{R}^{n}$ whose entries are all equal to 1 . If $g$ is homogeneous, we have for all $p \in \mathbb{R}^{n}, g^{*}(p) \geq \sup _{\lambda \in \mathbb{R}}\left(p \cdot \lambda 1_{n}-g\left(\lambda 1_{n}\right)\right)=\sup _{\lambda \in \mathbb{R}}\left(\lambda \sum_{1 \leq i \leq n} p_{i}-\lambda-g(0)\right)=$ $\sup _{\lambda \in \mathbb{R}} \lambda\left(\sum_{1 \leq i \leq n} p_{i}-1\right)-g(0)$. Hence, $g^{*}(p)<\infty$ implies that $\sum_{1 \leq i \leq n} p_{i}=1$. Similarly, if $g$ is monotone, we have $g^{*}(p) \geq \sup _{x \leq 0}(p \cdot x-g(x)) \geq \sup _{x \leq 0}(p \cdot x-g(0))$. Hence, $g^{*}(p)<\infty$ implies that $p_{1}, \ldots, p_{n} \geq 0$.

Corollary 2.2. If $g: \mathbb{R}^{n} \rightarrow \mathbb{R}$ is monotone, homogeneous, and convex, then, for all $v \in \mathbb{R}^{n}$, $g(v)=\sup _{p \in \operatorname{dom} g^{*}}\left(p \cdot v-g^{*}(p)\right)$, and the subdifferential

$$
\begin{aligned}
\partial g(v) \stackrel{\text { def }}{=}\left\{p \in \mathbb{R}^{n} \mid g(x)-g(v) \geq p \cdot(x-v), \forall x \in \mathbb{R}^{n}\right\} \\
=\left\{p \in \operatorname{dom} g^{*} \mid p \cdot v-g^{*}(p)=g(v)\right\}
\end{aligned}
$$

is a non-empty compact convex subset of $\mathscr{S}_{n}$.

Proof. Since $g$ is convex and takes only finite values, the subdifferential $\partial g(v)$ is non-empty for any $v \in \mathbb{R}^{n}$, and a subdifferential is trivially closed and convex. Moreover, $\partial g(v) \subset \operatorname{dom} g^{*}$ by definition of $\partial g(v)$, hence, by Proposition 2.1, $\partial g(v) \subset \mathscr{S}_{n}$. Finally, $\partial g(v)$ is compact as a closed subset of the compact set $\mathscr{S}_{n}$. 
Let $\mathscr{S}_{n n}$ denote the set of $n \times n$ (row-)stochastic matrices. For any $n \times n$ matrix $P$, we denote by $P_{1}, \ldots P_{n}$ the rows of $P$ and identify $P$ to $\left(P_{1}, \ldots, P_{n}\right)$, which amounts to identifying $\mathbb{R}^{n \times n}$ to $\left(\mathbb{R}^{1 \times n}\right)^{n}$ and $\mathscr{S}_{n n}$ to $\left(\mathscr{S}_{n}\right)^{n}$. If $f: \mathbb{R}^{n} \rightarrow \mathbb{R}^{n}$ is convex, we set, for all $v \in \mathbb{R}^{n}$,

$$
\partial f(v) \stackrel{\text { def }}{=}\left\{P \in \mathbb{R}^{n \times n} \mid f(x)-f(v) \geq P(x-v), \quad \forall x \in \mathbb{R}^{n}\right\} .
$$

If $f=\left(f_{1}, \ldots, f_{n}\right)$, the Fenchel transform of $f$ is the map $f^{*}: P \in \mathbb{R}^{n \times n} \mapsto$ $\left(f_{1}^{*}\left(P_{1}\right), \ldots, f_{n}^{*}\left(P_{n}\right)\right) \in(\mathbb{R} \cup\{+\infty\})^{n}$, and its domain is given by

$$
\operatorname{dom} f^{*}=\left\{P \in \mathbb{R}^{n \times n} \mid f^{*}(P) \in \mathbb{R}^{n}\right\}=\operatorname{dom} f_{1}^{*} \times \cdots \times \operatorname{dom} f_{n}^{*} .
$$

Of course

$$
\partial f(v)=\partial f_{1}(v) \times \cdots \times \partial f_{n}(v) \subset \operatorname{dom} f^{*} \subset \mathscr{S}_{n n} .
$$

when $f$ is monotone and homogeneous.

\subsection{Convex Rectangular Sets of Stochastic Matrices}

We say that a subset $\mathscr{P}$ of $\mathscr{S}_{n n}$ is rectangular if $\mathscr{P}=\mathscr{P}_{1} \times \cdots \times \mathscr{P}_{n}$, for some subsets $\mathscr{P}_{1}, \ldots, \mathscr{P}_{n}$ of $\mathscr{S}_{n}$. If $f: \mathbb{R}^{n} \rightarrow \mathbb{R}^{n}$ is a convex monotone homogeneous map, the domain of $f^{*}$ is rectangular, and the subdifferential $\partial f(v)$ of $f$ at any $v \in \mathbb{R}^{n}$ is rectangular. In this section, we extend the classical definition of graphs of stochastic matrices to the case of convex rectangular sets of stochastic matrices.

Let us first recall some classical notions. For all graphs $\mathscr{G}$, and nodes $i, j$ of $\mathscr{G}$, we say that $i$ has access to $j$, and we write $i \stackrel{*}{\rightarrow} j$, if there is a directed path from $i$ to $j$ in $\mathscr{G}$, or if $i=j$. We call classes of $\mathscr{G}$ the equivalence classes for the equivalence relation " $(i \stackrel{*}{\rightarrow} j)$ and $(j \stackrel{*}{\rightarrow} i)$ ". We call strongly connected component of $\mathscr{G}$, the restriction $\left.\mathscr{G}\right|_{C}$ of $\mathscr{G}$ to a class $C$ of $\mathscr{G}$, that is the subgraph of $\mathscr{G}$ with set of nodes $C$ and $\operatorname{arcs} i \rightarrow j$ when $(i \rightarrow j) \in \mathscr{G}$ and $i, j \in C$. A graph is strongly connected if it has only one class. A class $C$ of $\mathscr{G}$ is final if no nodes of $C$ have access to a node of the complement of $C$.

To any $n \times n$ nonnegative matrix $P$, we associate the (directed) $\operatorname{graph} \mathscr{G}(P)$ with nodes $1, \ldots, n$ and $\operatorname{arcs} i \rightarrow j$ when $P_{i j} \neq 0$. A matrix is irreducible if $\mathscr{G}(P)$ is strongly connected. Associating $\mathscr{G}(P)$ to $P$ allows us to use the graph vocabulary for nonnegative matrices, for instance the classes of $P$ are by definition the classes of $\mathscr{G}(P)$. If $P$ is a stochastic matrix, a class $C$ of $P$ is final if, and only if, the $C \times C$ submatrix of $P$ is stochastic. For any stochastic matrix $P$, we denote $\mathrm{N}^{\mathrm{f}}(P)$ the union of final classes of $P, \mathscr{C}^{\mathrm{f}}(P)$ the set of final classes of $P$ and $\mathscr{G}^{\mathrm{f}}(P)$ the subgraph of $\mathscr{G}(P)$ composed of the nodes and arcs of final classes of $P$, that is:

$$
\mathscr{G}^{\mathrm{f}}(P)=\bigcup_{F \text { fi nal class of } P} \mathscr{G}\left(P_{F F}\right) .
$$

Here and below, for all $n \times n$ matrices $P$ and subsets $I$ and $J$ of $\{1, \ldots, n\}$, we denote by $P_{I J}$ the $I \times J$ submatrix of $P$. If $\mathscr{P}$ is a rectangular set of stochastic matrices, we set

$$
\mathrm{N}^{\mathrm{f}}(\mathscr{P})=\bigcup_{P \in \mathscr{P}} \mathrm{N}^{\mathrm{f}}(P), \quad \mathscr{C}^{\mathrm{f}}(\mathscr{P})=\bigcup_{P \in \mathscr{P}} \mathscr{C}^{\mathrm{f}}(P), \quad \mathscr{G}^{\mathrm{f}}(\mathscr{P})=\bigcup_{P \in \mathscr{P}} \mathscr{G}^{\mathrm{f}}(P) .
$$


Hence, $\mathscr{G}^{\mathrm{f}}(\mathscr{P})$ has $\mathrm{N}^{\mathrm{f}}(\mathscr{P})$ as set of nodes, and $i \rightarrow j$ is an arc if there exists $P \in \mathscr{P}$ such that $P_{i j} \neq 0$ and $i$ and $j$ belong to the same final class of $P$. We say that a class $C \in \mathscr{C}^{\mathrm{f}}(P)$ is maximal if it is maximal for inclusion. We have:

Proposition 2.3. Let $\mathscr{P}$ be a convex rectangular set of stochastic matrices.

1. If $C, C^{\prime} \in \mathscr{C}^{\mathrm{f}}(\mathscr{P})$ and $C \cap C^{\prime} \neq \varnothing$, then, $C \cup C^{\prime} \in \mathscr{C}^{\mathrm{f}}(\mathscr{P})$;

2. The maximal elements of $\mathscr{C}^{\mathrm{f}}(\mathscr{P})$ are disjoint;

3. There is a matrix $P \in \mathscr{P}$ such that $\mathscr{G}^{\mathrm{f}}(P)=\mathscr{G}^{\mathrm{f}}(\mathscr{P})$. The final classes of $P$ are precisely the classes of $\mathscr{G}^{\mathrm{f}}(\mathscr{P})$.

4. The classes of $\mathscr{G}(\mathscr{P})$ are exactly the maximal elements of $\mathscr{C}^{\mathrm{f}}(\mathscr{P})$.

Proof. By assumption, $\mathscr{P}=\mathscr{P}_{1} \times \cdots \times \mathscr{P}_{n}$, where $\mathscr{P}_{1}, \ldots, \mathscr{P}_{n}$ are convex subsets of $\mathscr{S}_{n}$.

If $C, C^{\prime} \in \mathscr{C}^{\mathrm{f}}(\mathscr{P})$, then, we can find $P, P^{\prime} \in \mathscr{P}$ such that $C$ and $C^{\prime}$ are final classes of $P$ and $P^{\prime}$, respectively. Consider the matrix $P^{\prime \prime}$ defined by $P_{i}^{\prime \prime}=\left(P_{i}+P_{i}^{\prime}\right) / 2$ if $i \in C \cap C^{\prime}, P_{i}^{\prime \prime}=P_{i}$ if $i \in C \backslash C^{\prime}, P_{i}^{\prime \prime}=P_{i}^{\prime}$ if $i \in C^{\prime} \backslash C$, and $P_{i}^{\prime \prime}=P_{i}$ or $P_{i}^{\prime}$ for $i \in\{1, \ldots, n\} \backslash\left(C \cup C^{\prime}\right)$ (the choice has no importance). Since the $\mathscr{P}_{i}$ are convex, $P_{i}^{\prime \prime} \in \mathscr{P}_{i}$ for all $i$, and by rectangularity, $P^{\prime \prime} \in \mathscr{P}$. To prove the first assertion, it remains to check that $C \cup C^{\prime}$ is a final class of $P^{\prime \prime}$. By construction, the $\left(C \cup C^{\prime}\right) \times\left(C \cup C^{\prime}\right)$ submatrix of $P^{\prime \prime}$ has row-sum 1, hence, we have only to check that all the nodes of $C \cup C^{\prime}$ are mutually accessible in $\mathscr{G}\left(P^{\prime \prime}\right)$. By definition of $P^{\prime \prime}$, any path of $\mathscr{G}(P)$ (resp. $\mathscr{G}\left(P^{\prime}\right)$ ) that remains in $C$ (resp. $C^{\prime}$ ) is a path of $\mathscr{G}\left(P^{\prime \prime}\right)$. Picking any node $j \in C \cap C^{\prime}$, we see that there is a path from any $i \in C \cup C^{\prime}$ to $j$, and a path from $j$ to $i$, in $\mathscr{G}\left(P^{\prime \prime}\right)$, which shows the first assertion.

The second assertion follows readily from the first.

For the third point, using the method of the first part of the proof, we shall construct by convexification a matrix $Q \in \mathscr{P}$ such that $\mathscr{G}^{\mathrm{f}}(\mathscr{P})=\mathscr{G}^{\mathrm{f}}(Q)$. For each $\operatorname{arc} i \rightarrow j$ of $\mathscr{G}^{\mathrm{f}}(\mathscr{P})$, there is a matrix $P^{i j} \in \mathscr{P}$ such that $i$ and $j$ belong to the same final class $F^{i j}$ of $P^{i j}$, and $\left(P^{i j}\right)_{i j} \neq 0$. For each $k \in \mathrm{N}^{\mathrm{f}}(\mathscr{P})$, we consider $\mathscr{Q}_{k}=\left\{\left(P^{i j}\right)_{k} \mid(i \rightarrow j) \in \mathscr{G}^{\mathrm{f}}(\mathscr{P}), k \in F^{i j}\right\}$, and we build the stochastic matrix $Q$ whose $k$-th row is given by $Q_{k}=\left|\mathscr{Q}_{k}\right|^{-1}\left(\sum_{P \in \mathscr{Q}_{k}} P\right)$ for all $k \in \mathrm{N}^{\mathrm{f}}(\mathscr{P})$ (we denote by $|\cdot|$ the cardinality of a set), and $Q_{k}=$ any element of $\mathscr{P}_{k}$ if $k \notin \mathrm{N}^{\mathrm{f}}(\mathscr{P})$. By convexity and rectangularity of $\mathscr{P}, Q \in \mathscr{P}$, hence $\mathscr{G}^{\mathrm{f}}(Q) \subset \mathscr{G}^{\mathrm{f}}(\mathscr{P})$. By construction, each row of $Q$ with index in $\mathrm{N}^{\mathrm{f}}(\mathscr{P})$ has sum 1 , and $\mathscr{G}(Q)$ contains $\mathscr{G}^{\mathrm{f}}(\mathscr{P})$. Moreover, for any $\operatorname{arc}(k \rightarrow l)$ of $\mathscr{G}(Q)$ starting at $k \in \mathrm{N}^{\mathrm{f}}(\mathscr{P})$, there exists $(i \rightarrow j) \in \mathscr{G}(\mathscr{P})$ such that $\left(P^{i j}\right)_{k l}>0$ and $k \in F^{i j}$, hence $k$ and $l$ are in $F^{i j}$, which implies that $(k \rightarrow l) \in \mathscr{G}^{\mathrm{f}}(\mathscr{P})$. In particular there are no $\operatorname{arcs}$ in $\mathscr{G}(Q)$ going out from $\mathrm{N}^{\mathrm{f}}(\mathscr{P})$, and the restriction of $\mathscr{G}(Q)$ to $\mathrm{N}^{\mathrm{f}}(\mathscr{P})$ is equal to $\mathscr{G}^{\mathrm{f}}(\mathscr{P})$. All these remarks imply that $\mathscr{G}^{\mathrm{f}}(Q)$ contains $\mathscr{G}^{\mathrm{f}}(\mathscr{P})$, so $\mathscr{G}^{\mathrm{f}}(Q)=\mathscr{G}^{\mathrm{f}}(\mathscr{P})$.

Finally, any element $F$ of $\mathscr{C}^{\mathrm{f}}(\mathscr{P})$ (a fortiori, any maximal element) is such that two nodes of $F$ are connected by a directed path in $\mathscr{G}$ f $(\mathscr{P})$, so $F$ is included in a class of $\mathscr{G}^{\mathrm{f}}(\mathscr{P})$. Conversely, let $C$ be a class of $\mathscr{G}(\mathscr{P})$, and take the matrix $Q \in \mathscr{P}$ of the third point. Then $C$ is a final class of $Q$, hence $C \in \mathscr{C}^{\mathrm{f}}(\mathscr{P})$, so $C$ is included in a maximal element of $\mathscr{C}^{\mathrm{f}}(\mathscr{P})$. Since the classes of $\mathscr{G}^{\mathrm{f}}(\mathscr{P})$ are disjoint, we obtain the last point of the proposition. 
Example 2.4. Let $\operatorname{co}(X)$ denote the convex hull of a set $X$, and consider the convex rectangular set $\mathscr{P}=\mathscr{P}_{1} \times \mathscr{P}_{2} \times \mathscr{P}_{3} \subset \mathscr{S}_{33}$, with

$$
\begin{gathered}
\mathscr{P}_{1}=\operatorname{co}\{(1,0,0),(1 / 2,1 / 2,0),(1 / 2,0,1 / 2)\} \\
\mathscr{P}_{2}=\operatorname{co}\{(0,1,0),(2 / 3,1 / 3,0)\} \\
\mathscr{P}_{3}=\{(0,0,1)\} .
\end{gathered}
$$

Since the identity matrix $I$ belongs to $\mathscr{P},\{1\},\{2\},\{3\}$ all are elements of $\mathscr{C}^{\mathrm{f}}(\mathscr{P})$. Moreover, since

$$
\left(\begin{array}{ccc}
1 / 2 & 1 / 2 & 0 \\
2 / 3 & 1 / 3 & 0 \\
0 & 0 & 1
\end{array}\right) \in \mathscr{P},
$$

$\{1,2\}$ is also an element of $\mathscr{C}^{\mathrm{f}}(\mathscr{P})$. It is easy to see that these are the only elements of $\mathscr{C}^{\mathrm{f}}(\mathscr{P})$, so that its maximal elements for inclusion are $\{1,2\}$ and $\{3\}$. The final graph $\mathscr{G}^{\mathrm{f}}(\mathscr{P})$ of $\mathscr{P}$ is the following

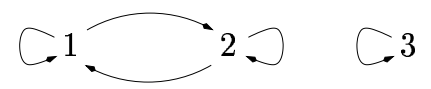

and its classes are $\{1,2\}$ and $\{3\}$. We shall give, at the end of Section 6 , an algorithm to determine the final graph of a convex rectangular sets $\mathscr{P}$ with a finite number of extremal points.

\subsection{Critical Graph of Convex Monotone Homogeneous Maps}

In the sequel, $f$ denotes a convex monotone homogeneous map that has an eigenvector. The associated eigenvalue will be denoted by $\lambda$. As pointed out in the introduction, we may assume $\lambda=0$, so that statements and proofs will often be given in this case. We shall prove:

Proposition 2.5. The sets $\mathrm{N}^{\mathrm{f}}(\partial f(v)), \mathscr{C}^{\mathrm{f}}(\partial f(v))$ and the graph $\mathscr{G}^{\mathrm{f}}(\partial f(v))$ all are independent of the choice of the eigenvector $v$ of $f$.

Thus, we shall simply write $\mathrm{N}^{\mathrm{c}}(f), \mathscr{C}^{\mathrm{c}}(f)$ and $\mathscr{G}^{\mathrm{c}}(f)$. We call critical nodes of $f$ the elements of $\mathrm{N}^{\mathrm{c}}(f)$, critical graph of $f$ the graph $\mathscr{G}^{\mathrm{c}}(f)$, and critical classes of $f$ the classes of $\mathscr{G}^{\mathrm{c}}(f)$. Combining Proposition 2.5 and Proposition 2.3, we get:

Corollary 2.6. 1. For any eigenvector $v$ of $f$, there is a matrix $P \in \partial f(v)$ such that $\mathscr{G}^{\mathrm{c}}(f)=$ $\mathscr{G}^{\mathrm{f}}(P)$. In particular, the final classes of $P$ are precisely the critical classes of $f$.

2. The critical classes of $f$ are exactly the maximal elements of $\mathscr{C}^{\mathrm{c}}(f)$.

The graph $\mathscr{G}^{\mathrm{c}}(f)$ generalizes the classical critical graph that appears in max-plus algebra. Let us

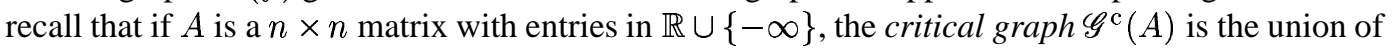
the circuits $\left(i_{1}, \ldots, i_{k}\right)$ that realize the maximum in the formula:

$$
\rho(A)=\max _{1 \leq k \leq n} \max _{i_{1}, \ldots, i_{k}} \frac{A_{i_{1} i_{2}}+\cdots+A_{i_{k} i_{1}}}{k} .
$$


If $A$ has at least one finite entry per row, the max-plus linear map $f_{A}$ with matrix $A,\left(f_{A}(x)\right)_{i}=$ $\max _{1 \leq j \leq n}\left(A_{i j}+x_{j}\right)$, sends $\mathbb{R}^{n}$ into $\mathbb{R}^{n}$. Of course, $f_{A}$ is monotone, homogeneous and convex. If $v \in \mathbb{R}^{n}$ is a max-plus eigenvector of $A$, with associated eigenvalue $\lambda$ (i.e. if $f_{A}(v)=\lambda+v$ ), we define the saturation graph of $A$ and $v, \operatorname{Sat}(A, v)$, as the union of $\operatorname{arcs} i \rightarrow j$ such that $\lambda+v_{i}=$ $A_{i j}+v_{j}$.

Proposition 2.7. If $A \in(\mathbb{R} \cup\{-\infty\})^{n \times n}$ has at least one finite entry per row, and if $v \in \mathbb{R}^{n}$ is an eigenvector of $A$, then $\mathscr{G}^{\mathrm{c}}\left(f_{A}\right)=\mathscr{G}^{\mathrm{c}}(A)$ coincides with the union of strongly connected components of $\operatorname{Sat}(A, v)$.

Proof. The fact that $\mathscr{G}^{\mathrm{c}}(A)$ coincides with the union of strongly connected components of $\operatorname{Sat}(A, v)$ is a consequence of the max-plus spectral theorem (see [BCOQ92, \$3.2.4 and 3.7]). The rest of the assertion follows from the identity $\left(\partial f_{A}(v)\right)_{i}=\operatorname{co}\left\{\delta_{j} \mid(i, j) \in \operatorname{Sat}(A, v)\right\}$, where $\delta_{j} \in \mathscr{S}_{n}$ denotes the Dirac probability measure at $j$. Indeed, from this, one deduces that, for any $P \in \partial f_{A}(v)$, $\mathscr{G}(P) \subset \operatorname{Sat}(A, v)$, so that $\mathscr{G}(P)$ is included in the union of strongly connected components of $\operatorname{Sat}(A, v)$, that is $\mathscr{G}^{\mathrm{c}}(A)$. Hence, $\mathscr{G}^{\mathrm{c}}\left(f_{A}\right) \subset \mathscr{G}^{\mathrm{c}}(A)$. Conversely, for any circuit $c$ in $\mathscr{G}^{\mathrm{c}}(A) \subset$ $\operatorname{Sat}(A, v)$, one can construct $P \in \partial f_{A}(v)$ such that $P_{i j}=1$ for any arc $i \rightarrow j$ of $c$. The circuit $c$, which is a final class of $P$, is included in $\mathscr{G}^{\mathrm{c}}\left(f_{A}\right)$. Thus, $\mathscr{G}^{\mathrm{c}}(A) \subset \mathscr{G}^{\mathrm{c}}\left(f_{A}\right)$.

(We shall extend this property to the stochastic control context in Proposition 7.2.)

Proposition 2.5 relies on classical facts of Perron-Frobenius theory. We first need the characterization of supports of invariant measures. Recall that an invariant measure of a stochastic matrix $P$ is a stochastic (row) vector $m$ such that $m P=m$. The set of non-zero entries of $m$ is the support of $m$. The following result is standard:

Proposition 2.8. Let $P$ be a $n \times n$ stochastic matrix. A subset $F$ of $\{1, \ldots, n\}$ is a union of final classes of $P$ if, and only if, $P$ has an invariant measure $m$ with support $F$.

The main ingredient of the proof of Proposition 2.5 is a classical property of super-harmonic vectors (a vector $v$ is super-harmonic for a stochastic matrix $P$ if $P v \leq v$ ), which is itself a consequence of the Perron-Frobenius theorem.

Lemma 2.9 (Discrete Maximum Principle). If $P$ is $a n \times n$ stochastic matrix, and if $P v \leq v$, then, the restriction of $v$ to any final class of $P$ is a constant vector, and $P v=v$ holds on the union $\mathrm{N}^{\mathrm{f}}(P)$ of the final classes of $P$. Moreover, the minimum of $v$ is attained on a final class.

Proof. Let $C$ denote a final class of $P$. It follows from Proposition 2.8 that there exists an invariant measure $m$ of $P$ with support $C$. We have, $0 \leq m(v-P v)=m v-m v=0$, and since $0=$ $m(v-P v)=\sum_{i \in C} m_{i}\left(v_{i}-(P v)_{i}\right)$ is a sum of nonnegative terms, we must have $v_{i}=(P v)_{i}$ for all $i \in C$. This means that the restriction of $v$ to $C$ is a right-eigenvector of the $C \times C$ submatrix of $P$. Since this submatrix is stochastic and irreducible, this eigenvector must be constant, which shows the first part of the lemma.

To show the second part, we introduce $F=\mathrm{N}^{\mathrm{f}}(P)$ and $T=\{1, \ldots, n\} \backslash F$. We denote by $Q$ the $T \times T$ submatrix of $P$, and by $w$ the restriction of $v$ to $T$. Let $\mu$ denote the minimum of the entries of $v$ restrained to $F$. Since $P 1_{n}=1_{n}, v-\mu \geq P(v-\mu)$, which allows us to assume 
that $\mu=0$. Then, $v \geq P v$ and $v \geq 0$ on $F$ yield $w \geq Q w$, and since $Q$ has nonnegative entries, $w \geq Q w \geq Q^{2} w \geq \ldots \geq Q^{k} w$, for all $k \geq 1$. Since $Q$ has spectral radius strictly less than 1 , we get $w \geq \lim _{k} Q^{k} w=0$.

We finally prove Proposition 2.5. Let $v, v^{\prime}$ denote two eigenvectors of $f$. Let $C \in \mathscr{C}^{\mathrm{f}}(\partial f(v))$ and let $P \in \partial f(v)$ for which $C$ is a final class. We get $v^{\prime}-v=f\left(v^{\prime}\right)-f(v) \geq P\left(v^{\prime}-v\right)$. It follows from Lemma 2.9 that $\left(v^{\prime}-v\right)_{i}=P_{i}\left(v^{\prime}-v\right)$ holds for all $i \in C$. But $f_{i}\left(v^{\prime}\right)-f_{i}(v)=$ $\left(v^{\prime}-v\right)_{i}=P_{i}\left(v^{\prime}-v\right)$ and $P_{i} \in \partial f_{i}(v)$ imply that $P_{i} \in \partial f_{i}\left(v^{\prime}\right)$ (indeed, $f_{i}(x)-f_{i}\left(v^{\prime}\right)=$ $f_{i}(x)-f_{i}(v)+f_{i}(v)-f_{i}\left(v^{\prime}\right) \geq P_{i}(x-v)-P_{i}\left(v^{\prime}-v\right) \geq P_{i}\left(x-v^{\prime}\right)$ holds for all $\left.x \in \mathbb{R}^{n}\right)$. Now, pick any $Q \in \partial f\left(v^{\prime}\right)$, and consider the matrix $R$ such that $R_{i}=P_{i}$ for all $i \in C$ and $R_{i}=Q_{i}$ if $i \in\{1, \ldots, n\} \backslash C$. By rectangularity of $\partial f\left(v^{\prime}\right), R \in \partial f\left(v^{\prime}\right)$. Since $C$ is a final class of $R$, $C \in \mathscr{C}^{\mathrm{f}}\left(\partial f\left(v^{\prime}\right)\right)$. This shows that $\mathscr{C}^{\mathrm{f}}(\partial f(v)) \subset \mathscr{C}^{\mathrm{f}}\left(\partial f\left(v^{\prime}\right)\right)$. Since $R_{C C}$ and $P_{C C}$ coincide, this also shows that $\mathscr{G}^{\mathrm{f}}(\partial f(v)) \subset \mathscr{G}^{\mathrm{f}}\left(\partial f\left(v^{\prime}\right)\right)$, and thus $\mathrm{N}^{\mathrm{f}}(\partial f(v)) \subset \mathrm{N}^{\mathrm{f}}\left(\partial f\left(v^{\prime}\right)\right)$.

Example 2.10. Let $f: \mathbb{R}^{3} \rightarrow \mathbb{R}^{3}$,

$$
f(x)=\left(\begin{array}{c}
x_{1} \vee \frac{1}{2}\left(x_{1}+x_{2}\right) \vee\left(-3+x_{3}\right) \vee \frac{1}{2}\left(x_{1}+x_{3}\right) \\
x_{2} \vee \frac{1}{3}\left(2 x_{1}+x_{2}\right) \vee\left(-1+x_{1}\right) \\
x_{3} \vee\left(-2+x_{1}\right)
\end{array}\right),
$$

which is such that $f(0)=0$. We have $\partial f(0)=\mathscr{P}$, with $\mathscr{P}$ defined in Example 2.4 (that is $\partial f_{i}(0)=$ $\mathscr{P}_{i}$ for $\left.i=1,2,3\right)$. So, the critical graph $\mathscr{G}^{\mathrm{c}}(f)$ of $f$ is exactly the final graph of $\mathscr{P}$ shown in Example 2.4, and the critical classes of $f$ are $\{1,2\}$ and $\{3\}$. Let us illustrate the fact that $\mathscr{G}^{\mathrm{f}}(\partial f(v))$ is independent of the choice of the eigenvector $v$ by considering the eigenvector $v=(0,0,-2)$. By comparison with $\partial f(0)$, only the first and third rows of $\partial f(v)$ are changed, namely $\partial f_{1}(v)=$ $\operatorname{co}\{(1,0,0),(1 / 2,1 / 2,0)\}$ and $\partial f_{3}(v)=\operatorname{co}\{(0,0,1),(1,0,0)\}$. Although $\partial f(v) \neq \partial f(0)$, the final classes of the matrices $P \in \partial f(v)$ are the same as the ones of the matrices $P \in \partial f(0)=\mathscr{P}$.

\section{Structure of Eigenspaces}

In order to make more apparent the proof idea, we first show a simple result.

Theorem 3.1. The eigenvector of a convex monotone homogeneous map with a unique critical class is unique, up to an additive constant.

Proof. Let $C$ denote the critical class of $f$, and let $v, v^{\prime}$ be two eigenvectors of $f$. Using Assertion 1 of Corollary 2.6, we get matrices $P \in \partial f(v)$ and $P^{\prime} \in \partial f\left(v^{\prime}\right)$ such that $C$ is the unique final class of $P$ and $P^{\prime}$. Since $P \in \partial f(v), v^{\prime}-v=f\left(v^{\prime}\right)-f(v) \geq P\left(v^{\prime}-v\right)$, hence, by Lemma 2.9, $v^{\prime}-v$ is constant on $C$, and it attains its minimum in $C$. Exchanging the roles of $v^{\prime}$ and $v$, we see that $v^{\prime}-v$ attains its maximum in $C$. Therefore, $v^{\prime}-v$ is a constant vector.

Example 3.2. Let us use Theorem 3.1 to show that the map $f: \mathbb{R}^{3} \rightarrow \mathbb{R}^{3}$,

$$
f(x)=\left(\begin{array}{c}
\frac{1}{2}\left(x_{1}+x_{2}\right) \\
\log \left(e^{x_{2}}+8 e^{x_{3}}\right) \\
x_{1} \vee\left(-1+x_{3}\right)
\end{array}\right)
$$


has a unique eigenvector, up to an additive constant. Since the graph $\mathscr{G}(f)$ of $f$, which is equal to

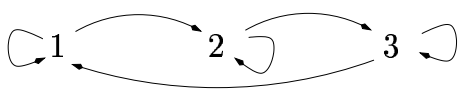

is strongly connected, we known, thanks to [GG01, Theorem 2], that $f$ has an eigenvector. Indeed, $f(v)=\lambda+v$, where $\lambda=\log 2$, and $v=(\log 2,3 \log 2,0)$. We have

$$
\partial f(v)=\left\{f^{\prime}(v)\right\} \text { where } f^{\prime}(v)=\left(\begin{array}{ccc}
1 / 2 & 1 / 2 & 0 \\
0 & 1 / 2 & 1 / 2 \\
1 & 0 & 0
\end{array}\right)
$$

Thus, the critical graph $\mathscr{G}^{\mathrm{c}}(f)=\mathscr{G}^{\mathrm{f}}\left(f^{\prime}(v)\right)=\mathscr{G}\left(f^{\prime}(v)\right)$ is

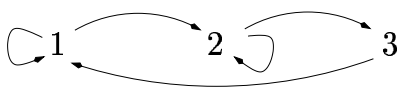

(only the arc $3 \rightarrow 3$ vanished, by comparison with $\mathscr{G}(f)$ ). Since $\mathscr{G}^{\mathrm{c}}(f)$ is strongly connected, $f$ has a unique critical class (namely $\{1,2,3\}$ ), and, by Theorem 3.1, the eigenvector of $f$ is unique (up to an additive constant). Equivalently, the map $F:\left(\mathbb{R}_{+}^{*}\right)^{3} \rightarrow\left(\mathbb{R}_{+}^{*}\right)^{3}, F=\exp \circ f \circ \log$,

$$
F(x)=\left(\begin{array}{c}
\sqrt{x_{1} x_{2}} \\
x_{2}+8 x_{3} \\
x_{1} \vee\left(x_{3} / e\right)
\end{array}\right)
$$

has a unique (multiplicative) eigenvector, up to a constant, namely, $(2,8,1)$.

To extend Theorem 3.1 to the case of several critical classes, we shall use the following fundamental observation, which establishes the existence of a monotone homogeneous projector $(-\lambda+f)^{\omega}$ from the super-eigenspace of $f, \mathscr{E}+(f)=\left\{x \in \mathbb{R}^{n} \mid f(x) \leq \lambda+x\right\}$, to the eigenspace $\mathscr{E}(f)=\left\{x \in \mathbb{R}^{n} \mid f(x)=\lambda+x\right\}$.

Lemma 3.3 (Spectral Projector). If $f: \mathbb{R}^{n} \rightarrow \mathbb{R}^{n}$ is a convex monotone homogeneous map with eigenvalue 0 , and if $z \in \mathbb{R}^{n}$ is such that $f(z) \leq z$, then

$$
f^{\omega}(z) \stackrel{\text { def }}{=} \lim _{k \rightarrow \infty} f^{k}(z)
$$

is an eigenvector of $f$, which coincides with $z$ on the union $\mathrm{N}^{\mathrm{c}}(f)$ of critical classes of $f$. The map $f^{\omega}: \mathscr{E}^{+}(f) \rightarrow \mathscr{E}(f)$ is monotone, homogeneous, convex, onto, and satisfies $\left(f^{\omega}\right)^{2}=f^{\omega}$.

Proof. By monotonicity of $f$, the sequence $z_{k}$ defined by $z_{0}=z, z_{k}=f\left(z_{k-1}\right)$ for $k \geq 1$, is nonincreasing. Picking any eigenvector $v$ of $f$ and using the nonexpansiveness of $f$, we get $\left|z_{k}-v\right|=$ $\left|f^{k}\left(z_{0}\right)-f^{k}(v)\right| \leq\left|z_{0}-v\right|$. Therefore, the sequence $z_{k}$ which is bounded and nonincreasing converges to a limit $f^{\omega}(z)$, which by continuity of $f$ is an eigenvector of $f$. To show that $f^{\omega}(z)$ coincides with $z$ on $C=\mathrm{N}^{\mathrm{c}}(f)$, we observe that, for all $u \in \mathbb{R}^{n}$,

$$
f(u) \leq u \Longrightarrow f(u)=u \text { on } C .
$$


Indeed, arguing as in the proof of Theorem 3.1, we can find, by Corollary 2.6, a matrix $P \in \partial f(v)$ which has $C$ as union of final classes. Then, $u-v \geq f(u)-f(v) \geq P(u-v)$, and by Lemma 2.9, $u-v=P(u-v)$ on $C$, which implies that $u-v=f(u)-f(v)$ on $C$, and since $v=f(v)$, we get (7). Applying (7) to the inequalities $f\left(z_{0}\right) \leq z_{0}, f\left(z_{1}\right) \leq z_{1}, \ldots$, we get $z_{1}=f\left(z_{0}\right)=z_{0}$ on $C, z_{2}=f\left(z_{1}\right)=z_{1}$ on $C, \ldots$, hence $z_{k}=z_{0}$ on $C$ for all $k \geq 0$, which implies that $f^{\omega}(z)=$ $\lim _{k} z_{k}=z$ on $C$. Finally, $f^{\omega}: \mathscr{E}^{+}(f) \rightarrow \mathscr{E}(f)$ is onto since $f^{\omega}(x)=x$ when $x \in \mathscr{E}(f)$, and $f^{\omega}$, which is a pointwise limit of the monotone, homogeneous, and convex maps $f^{k}$ is also monotone, homogeneous, and convex.

The super-eigenspace $\mathscr{E}^{+}(f)$ will be useful in the subsequent proofs, because $\mathscr{E}^{+}(f)$ is a "more regular" object than $\mathscr{E}(f)$. In particular, thanks to the monotonicity of $f, \mathscr{E}^{+}(f)$ is an infsubsemilattice of $\left(\mathbb{R}^{n}, \leq\right)$, and, when $f$ is convex, $\mathscr{E}^{+}(f)$ is convex. If $C$ is any subset of $\{1, \ldots, n\}$, we denote by $\mathbb{R}^{C}$ the set of vectors indexed by the elements of $C$ and by $\mathrm{r}_{C}$ the restriction map $\mathbb{R}^{n} \rightarrow \mathbb{R}^{C}, \mathrm{r}_{C}(x)_{i}=x_{i}$, for all $i \in C$.

Theorem 3.4. Let $C_{1}, \ldots, C_{s}$ denote the critical classes of a convex monotone homogeneous map $f$, and let $C=C_{1} \cup \ldots \cup C_{s}=\mathrm{N}^{\mathrm{c}}(f)$. Then, any two eigenvectors $v$, $v^{\prime}$ of $f$ satisfy:

1. $v-v^{\prime}$ is constant on each $C_{i}$;

2. if $v=v^{\prime}$ on $C$, then $v=v^{\prime}$.

\section{Moreover,}

3. the restriction $\mathrm{r}_{C}$ sends bijectively $\mathscr{E}(f)$ to a convex inf-subsemilattice of $\mathbb{R}^{C}$, denoted by $\mathscr{E}^{\mathrm{c}}(f)$, and the inverse map $\mathrm{r}_{C}^{-1}: \mathscr{E}^{\mathrm{c}}(f) \rightarrow \mathscr{E}(f)$ is monotone and homogeneous.

Proof. Let $v, v^{\prime}$ be two eigenvectors of $f$. Arguing as in the proof of Theorem 3.1, we get two matrices $P \in \partial f(v)$ and $P^{\prime} \in \partial f\left(v^{\prime}\right)$ which both have exactly the final classes $C_{1}, \ldots, C_{s}$. Since $v^{\prime}-v \geq P\left(v^{\prime}-v\right)$, we get from Lemma 2.9 that $v^{\prime}-v$ is constant on each final class $C_{i}$, which proves the first assertion. Moreover, if $v^{\prime}-v \geq 0$ on $C$, the vector $v^{\prime}-v$, which, by Lemma 2.9, attains its minimum on a final class, must be nonnegative. By symmetry, if $v-v^{\prime} \geq 0$ on $C, v-v^{\prime}$ must be nonnegative. Thus, $v=v^{\prime}$ on $C$ implies $v=v^{\prime}$, which shows the second assertion of the theorem. This assertion implies that $\mathbf{r}_{C}$ is injective $\mathscr{E}(f) \rightarrow \mathscr{E}^{\mathscr{C}}(f)$. The inverse map $\mathbf{r}_{C}^{-1}$ is trivially homogeneous. It is also monotone: if $w^{\prime}=\mathrm{r}_{C}\left(v^{\prime}\right) \geq w=\mathrm{r}_{C}(v), v^{\prime}-v \geq 0$ on $C$, and we just saw that this implies that $v^{\prime}-v \geq 0$. Thus, $w^{\prime} \geq w \Longrightarrow \mathrm{r}_{C}^{-1}\left(w^{\prime}\right) \geq \mathrm{r}_{C}^{-1}(w)$.

Take now $v, v^{\prime}$ two eigenvectors of $f$, and assume that the eigenvalue of $f$ is 0 . By monotonicity of $f, f\left(v \wedge v^{\prime}\right) \leq v \wedge v^{\prime}$, and by Lemma 3.3, $f^{\omega}\left(v \wedge v^{\prime}\right)$ is an eigenvector which coincides with $v \wedge v^{\prime}$ on $C$. This shows that $\mathscr{E}^{c}(f)$, equipped with the ordering $\leq$, is an inf-semilattice, in which the greatest lower bound coincides with that of $\mathbb{R}^{C}$.

Finally, let $0<\alpha<1$, and $\alpha^{\prime}=1-\alpha$. We have, by convexity of $f, f\left(\alpha v+\alpha^{\prime} v^{\prime}\right) \leq$ $\alpha f(v)+\alpha^{\prime} f\left(v^{\prime}\right)=\alpha v+\alpha^{\prime} v^{\prime}$, and arguing as for $v \wedge v^{\prime}$, we derive from this that $\mathscr{E}^{c}(f)$ is convex.

Of course, Theorem 3.4 implies that the eigenspace $\mathscr{E}(f)$ is connected $(\mathscr{E}(f)$ is the image of the convex set $\mathscr{E}^{\mathfrak{C}}(f)$ by the continuous map $\left.\mathrm{r}_{C}^{-1}\right)$. More generally, Bruck [Bru73] proved that the set of 
fixed points of a nonexpansive map in a Banach space is a retract of the whole space, and, a fortiori, is connected. In the special case of convex monotone homogeneous maps, we have more, since Theorem 3.4 shows that the eigenspace $\mathscr{E}(f)$ is isomorphic (by monotone homogeneous bijections) to the convex set $\mathscr{E}^{\mathrm{c}}(f)$. This makes the following definition natural.

Definition 3.5. The dimension $\operatorname{dim} \mathscr{E}(f)$ of the eigenspace $\mathscr{E}(f)$ of a convex monotone homogeneous map $f$ is the dimension (as a convex set) of its restriction $\mathscr{E}^{c}(f)$ to the union of critical classes.

(Recall that the dimension of a convex set is defined as the dimension of its affine hull). A perhaps more convenient way to describe the geometry of $\mathscr{E}(f)$ is to introduce the notion of section, which is any set $S \subset \mathrm{N}^{\mathrm{c}}(f)$ which meets each critical class at exactly one point. We denote by $\mathrm{m}(f)$ the number of critical classes of $f$. Then, paraphrasing Theorem 3.4, we get:

Corollary 3.6. Let $f$ denote a convex monotone homogeneous map $f$ with section $S$. Then, $\mathscr{E}^{\mathrm{c}}(f)$ and $\mathbf{r}_{S}(\mathscr{E}(f))$ both are convex sets of dimension $\operatorname{dim} \mathscr{E}(f) \leq \mathrm{m}(f)$.

A natural question is to characterize the dimension of $\mathscr{E}(f)$. We shall see that the equality $\operatorname{dim} \mathscr{E}(f)=\mathrm{m}(f)$ holds, except in "singular" cases. Let us first exhibit a regular case.

Example 3.7. For the map $f$ given in (6), we saw in Example 2.10 that the critical classes of $f$ are $\{1,2\}$ and $\{3\}$. In particular, $\mathrm{m}(f)=2$. Since $v=0$ is an eigenvector, the first statement of Theorem 3.4 shows that any eigenvector is constant on $\{1,2\}$. Then, an elementary computation shows that $\mathscr{E}(f)=\left\{x \in \mathbb{R}^{3} \mid f(x)=x\right\}=\left\{x \in \mathbb{R}^{3} \mid x_{1}=x_{2} \geq x_{3} \geq-2+x_{1}\right\}$. Thus $\operatorname{dim} \mathscr{E}(f)=2=\mathrm{m}(f)$.

Example 3.8. It is instructive to apply Theorem 3.4 or Theorem 3.1 to the case of the map $f(x)=$ $\log M \exp (x)$, i.e., $f_{i}(x)=\log \left(\sum_{j} M_{i j} \exp \left(x_{j}\right)\right)$, where $M$ is an irreducible nonnegative matrix $M$. We have $\partial f(x)=\{P(x)\}$, where $P(x)=f^{\prime}(x)$ is the $n \times n$ matrix with entries $P(x)_{i j}=$ $\left(\sum_{k} M_{i k} \exp \left(x_{k}\right)\right)^{-1} M_{i j} \exp \left(x_{j}\right)$. Since $M$ is irreducible, $P(x)$ is irreducible, for all values of $x$. Therefore, $f$ has a unique critical class, and Theorem 3.1 tells that its eigenvector is unique, up to an additive constant, or, equivalently, that the matrix $M$ has a unique positive eigenvector, up to a multiplicative constant. This is a (complicated) way to derive the uniqueness part of PerronFrobenius theorem for irreducible nonnegative matrices, from the Perron-Frobenius theorem for irreducible stochastic matrices.

The next example illustrates the difference between regular and singular cases.

Example 3.9. Let $h$ denote any convex map $\mathbb{R} \rightarrow \mathbb{R}$ whose subgradients are between 0 and 1 . The map $f: \mathbb{R}^{2} \rightarrow \mathbb{R}^{2}$,

$$
f(x)=\left(\begin{array}{c}
x_{1}+h\left(x_{2}-x_{1}\right) \\
x_{2}+h\left(x_{1}-x_{2}\right)
\end{array}\right)
$$

is monotone, homogeneous, convex, and $f(0)=h(0)+0$, which means that 0 is an eigenvector of $f$ for the eigenvalue $h(0)$. If $(h(t)=h(-t) \Longrightarrow t=0)$, then 0 is the unique eigenvector of $f$, up to an additive constant. This condition is satisfied by the maps $t \mapsto \log \left(1+e^{t}\right)$ and $t \mapsto 0 \vee t$. It is also satisfied by the Moreau-Yoshida regularization, $h_{\epsilon}$, of $t \mapsto 0 \vee t$, which, for all $\epsilon>0$, is defined by $h_{\epsilon}(t)=0$ for $t \leq 0, h_{\epsilon}(t)=t^{2} / 2 \epsilon$ for $0 \leq t \leq \epsilon$, and $h_{\epsilon}(t)=t-\epsilon / 2$ for $t \geq \epsilon$. 
Let $g, k$, and $k^{\epsilon}$ denote the maps $f$ obtained by replacing $h$ by $t \mapsto \log \left(1+e^{t}\right), t \mapsto 0 \vee t$, and $h_{\epsilon}$ in (8), respectively. We have $\partial g_{1}(0)=\left\{\left(g_{1}\right)^{\prime}(0)\right\}=\{(1 / 2,1 / 2)\}, \partial k_{1}(0)=\operatorname{co}\{(1,0),(0,1)\}$, and $\partial k_{1}^{\epsilon}(0)=\left\{\left(k_{1}^{\epsilon}\right)^{\prime}(0)\right\}=\{(1,0)\}$. Using the symmetry between the first and the second coordinate of $f$, we get the following critical graphs

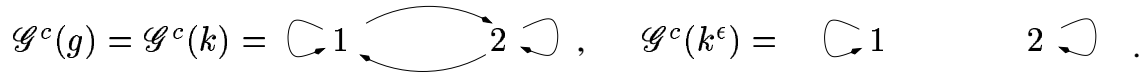

Thus, $\operatorname{dim} \mathscr{E}(g)=\mathrm{m}(g)=1, \operatorname{dim} \mathscr{E}(k)=\mathrm{m}(k)=1$, but $\operatorname{dim} \mathscr{E}\left(k^{\epsilon}\right)=1<\mathrm{m}\left(k^{\epsilon}\right)=2$. This discrepancy should be intuitively clear by looking at the following graphs:
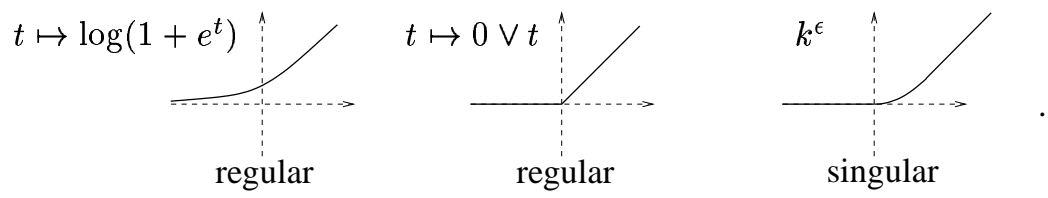

In the case of $f=k^{\epsilon}$, Theorem 3.4 fails to characterize the dimension of $\mathscr{E}(f)$ because the subdifferential of $f$ does not give enough information on the local behavior of $f$ near the eigenspace. In such cases, one needs to consider terms of higher order in the local expansion of $f$ to establish the uniqueness of the eigenvector. However, we shall see in section 6 that the equality $\operatorname{dim} \mathscr{E}(f)=\mathrm{m}(f)$ does hold when $f$ is piecewise affine.

The following last example shows that $\mathscr{E}(f)$ need not be convex or an inf-subsemilattice of $\mathbb{R}^{n}$, even if its restriction $\mathscr{E}^{\mathrm{c}}(f)$ is a convex inf-subsemilattice of $\mathbb{R}^{C}$.

Example 3.10. Consider $f: \mathbb{R}^{3} \rightarrow \mathbb{R}^{3}$,

$$
f(x)=\left(\begin{array}{c}
x_{2} \vee x_{3} \\
x_{2} \\
x_{3}
\end{array}\right) .
$$

We have $\mathscr{E}(f)=\left\{\left(x_{2} \vee x_{3}, x_{2}, x_{3}\right) \mid x_{2}, x_{3} \in \mathbb{R}\right\}, f$ has two critical classes $C_{1}=\{2\}, C_{2}=\{3\}$, so $\operatorname{dim} \mathscr{E}(f)=\mathrm{m}(f)=2$. Let $u=(1,1,0), v=(1,0,1)$. Since $u, v \in \mathscr{E}(f)$ but $(u+v) / 2=$ $(1,1 / 2,1 / 2) \notin \mathscr{E}(f), \mathscr{E}(f)$ is not convex, and since $u \wedge v=(1,0,0) \notin \mathscr{E}(f), \mathscr{E}(f)$ is not an inf-subsemilattice of $\mathbb{R}^{C}$.

Finally, we note that fixed points sets of monotone nonexpansive maps $\mathbb{R}^{n} \rightarrow \mathbb{R}^{n}$ are always lattices: the interesting lattice statement in assertion 3 of Theorem 3.4 is that the inf law of $\mathscr{E}^{c}(f)$ coincides with the usual inf law in $\mathbb{R}^{C}$.

Proposition 3.11. Let $f: \mathbb{R}^{n} \rightarrow \mathbb{R}^{n}$ denote a monotone map that is nonexpansive (for any norm) and that has a fixed point. Then, the fixed point set $\mathscr{E}(f)=\left\{x \in \mathbb{R}^{n} \mid f(x)=x\right\}$, equipped with the standard order relation, is a lattice, in which the sup and inf laws, $\vee_{f}$ and $\wedge_{f}$, respectively, are given by

$$
x \vee_{f} y=\lim _{k \rightarrow \infty} f^{k}(x \vee y), \quad x \wedge_{f} y=\lim _{k \rightarrow \infty} f^{k}(x \wedge y) .
$$


Proof. If $x, y \in \mathscr{E}(f)$, then by monotonicity of $f, f(x \wedge y) \leq f(x) \wedge f(y)=x \wedge y$. Arguing as at the beginning of the proof of Lemma 3.3, we get that the limit $w=\lim _{k \rightarrow \infty} f^{k}(x \wedge y)$ exists and satisfies $f(w)=w, w \leq x$, and $w \leq y$. Moreover, if $z$ is an arbitrary element in $\mathscr{E}(f)$ such that $z \leq x$, and $z \leq y$, we have $z=f(z)=\cdots=f^{k}(z) \leq f^{k}(x \wedge y)$ for all $k$, so that $z \leq w$. This shows that $w=\lim _{k \rightarrow \infty} f^{k}(x \wedge y)=x \wedge_{f} y$. The dual argument shows the first equality in (9).

\section{Critical Graph of $f^{k}$}

In this section, we establish the following result which is central in the proof of the cyclicity theorem. If $\mathscr{G}$ is a graph, we call $\mathscr{G}^{k}$ the graph with same nodes as $\mathscr{G}$ and $\operatorname{arcs}(i \rightarrow j)$ when there is a directed path $i \rightarrow i_{1} \rightarrow \cdots \rightarrow i_{k}=j$ with length $k$ in $\mathscr{G}$.

Theorem 4.1. Let $f$ denote a convex monotone homogeneous map $\mathbb{R}^{n} \rightarrow \mathbb{R}^{n}$ that has an eigenvector. Then, for all $k \geq 1, \mathscr{G}^{\mathrm{c}}\left(f^{k}\right)=\mathscr{G}^{\mathrm{c}}(f)^{k}$, in particular $\mathrm{N}^{\mathrm{c}}\left(f^{k}\right)=\mathrm{N}^{\mathrm{c}}(f)$.

The proof of Theorem 4.1 needs tools and results, of independent interest, which involve onesided directional derivatives, additive recession functions, and a nonlinear generalization of invariant measures. Let us first show the linear version of Theorem 4.1.

Proposition 4.2. If $P$ is a stochastic matrix, then, for all $k \geq 1, \mathscr{G}^{\mathrm{f}}\left(P^{k}\right)=\mathscr{G}^{\mathrm{f}}(P)^{k}$, in particular $\mathrm{N}^{\mathrm{f}}\left(P^{k}\right)=\mathrm{N}^{\mathrm{f}}(P)$.

Proof. For all nonnegative matrices $P$, we have $\mathscr{G}\left(P^{k}\right)=\mathscr{G}(P)^{k}$. Since $\mathscr{G}^{\mathrm{f}}(P)$ is equal to $\mathscr{G}(Q)$, where $Q$ is the restriction of $P$ to $\mathrm{N}^{\mathrm{f}}(P)$ and since the restriction of $P^{k}$ to $\mathrm{N}^{\mathrm{f}}(P)$ is equal to $Q^{k}$ (by definition of final classes), it is enough to prove that $\mathrm{N}^{\mathrm{f}}\left(P^{k}\right)=\mathrm{N}^{\mathrm{f}}(P)$.

To show this, we shall use the following consequence of Proposition 2.8: for any stochastic matrix $P$, the support of an invariant measure $m$ of $P$ is a subset of $\mathrm{N}^{\mathrm{f}}(P)$, and there exists an invariant measure $m$ of $P$ with support $\mathrm{N}^{\mathrm{f}}(P)$.

Consider first an invariant measure $m$ of $P$ with support $\mathrm{N}^{\mathrm{f}}(P)$. The vector $m$ is also an invariant measure of $P^{k}$, which implies that its support $\mathrm{N}^{\mathrm{f}}(P)$ is included in $\mathrm{N}^{\mathrm{f}}\left(P^{k}\right)$. Conversely, consider an invariant measure $m$ of $P^{k}$ with support $\mathrm{N}^{\mathrm{f}}\left(P^{k}\right)$. We get $m\left(I+\cdots+P^{k-1}\right) P=m\left(P+\cdots+P^{k}\right)=$ $m\left(I+\cdots+P^{k-1}\right)$, so $m_{k}=\frac{1}{k} m\left(I+\cdots+P^{k-1}\right)$ is an invariant measure of $P$. Hence, the support of $m_{k}$ is included in $\mathrm{N}^{\mathrm{f}}(P)$. Since $m_{k} \geq \frac{1}{k} m \geq 0$, due to the nonnegativeness of $P$ and $m$, the support of $m_{k}$ contains that of $m$, which leads to $\mathrm{N}^{\mathrm{f}}\left(P^{k}\right) \subset \mathrm{N}^{\mathrm{f}}(P)$.

For any convex map $f: \mathbb{R}^{n} \rightarrow \mathbb{R}$ and any $v \in \mathbb{R}^{n}$, we denote by $f_{v}^{\prime}(y)$ the (one-sided) directional derivative of $f$ at $v$ with respect to $y$ :

$$
f_{v}^{\prime}(y)=\lim _{\varepsilon \rightarrow 0^{+}} \frac{f(v+\varepsilon y)-f(v)}{\varepsilon} .
$$

The map $f_{v}^{\prime}$ is well-defined, finite, convex, multiplicatively homogeneous, and

$$
f_{v}^{\prime}(y)=\sup _{p \in \partial f(v)} p \cdot y
$$

RR n ${ }^{\circ} 4273$ 
see [Roc70, Theorems 23.1 and 23.4]. When $f$ is monotone and additively homogeneous, so does $f_{v}^{\prime}$. In the sequel, we shall say that a map is bihomogeneous if it is both additively and multiplicatively homogeneous.

The definition and properties of $f_{v}^{\prime}$ can be extended to convex maps $f: \mathbb{R}^{n} \rightarrow \mathbb{R}^{m}$, with $f_{v}^{\prime}=\left(\left(f_{1}\right)_{v}^{\prime}, \ldots,\left(f_{m}\right)_{v}^{\prime}\right): \mathbb{R}^{n} \rightarrow \mathbb{R}^{m}$. We have the following chain rules:

Lemma 4.3. Let $f: \mathbb{R}^{n} \rightarrow \mathbb{R}^{m}$ and $g: \mathbb{R}^{m} \rightarrow \mathbb{R}^{\ell}$ be two convex maps. Assume that $g$ is monotone. Then, $g \circ f: \mathbb{R}^{n} \rightarrow \mathbb{R}^{\ell}$ is convex, and, for all $v \in \mathbb{R}^{n}$,

$$
\begin{aligned}
(g \circ f)_{v}^{\prime} & =g_{f(v)}^{\prime} \circ f_{v}^{\prime}, \\
\partial(g \circ f)(v) & =\operatorname{co}(\partial g(f(v)) \partial f(v)) .
\end{aligned}
$$

(Recall that co denotes the convex hull. In (12), $\partial g(f(v)) \partial f(v) \stackrel{\text { def }}{=}\{P Q \mid P \in \partial g(f(v)), Q \in$ $\partial f(v)\}$.

Proof. The convexity of $g \circ f$ is immediate, and Eqn (11) follows easily from the fact that any finite convex function is locally Lipschitz continuous.

To show (12), we first note that if $D$ is a rectangular subset of $\mathbb{R}^{m}$, i.e. if $D$ is of the form $D_{1} \times \cdots \times D_{m}$, and if $h: \mathbb{R}^{m} \rightarrow \mathbb{R}^{p}$ is monotone and satisfies $h\left(\lim _{k} x_{k}\right)=\lim _{k} h\left(x_{k}\right)$ for all nondecreasing sequences $x_{k}$, then,

$$
h\left(\sup _{d \in D} d\right)=\sup _{d \in D} h(d) .
$$

Indeed, the inequality $\geq$ in (13) follows from the monotonicity of $h$, and, thanks to the rectangularity of $D$, we can find a nondecreasing sequence $d_{k} \in D$ such that $\sup _{d \in D} d=\lim _{k} d_{k}$, hence, $h\left(\sup _{d \in D} d\right)=h\left(\lim _{k} d_{k}\right)=\lim _{k} h\left(d_{k}\right) \leq \sup _{d \in D} h(d)$, which shows (13).

Applying (13) to $D=\partial f(v) y$ and $h(x)=P x$, with $P \in \partial g(f(v))$, we get from (11) and (10),

$$
\begin{aligned}
(g \circ f)_{v}^{\prime}(y)=g_{f(v)}^{\prime} \circ f_{v}^{\prime}(y) & =\sup _{P \in \partial g(f(v))} P\left(\sup _{Q \in \partial f(v)} Q y\right) \\
& =\sup _{R \in \partial g(f(v)) \partial f(v)} R y .
\end{aligned}
$$

Let us denote by $\delta_{X}^{*}: \mathbb{R}^{n} \rightarrow \mathbb{R} \cup\{+\infty\}$ the support function of a subset $X \subset \mathbb{R}^{n}$, which is defined by $\delta_{X}^{*}(p)=\sup _{x \in X} p \cdot x$. We first assume that $\ell=1$. Then, (14) and property (10) show that $(g \circ f)_{v}^{\prime}=\delta_{\partial(g \circ f)(v)}^{*}=\delta_{\partial g(f(v)) \partial f(v)}^{*}$. By Legendre-Fenchel duality, two subsets of $\mathbb{R}^{n}$ have the same support function, if, and only if, they have the same closed convex hull (see [Roc70, Corollary 13.1.1]). Since $\partial(g \circ f)(v)$ is closed and convex, $\partial(g \circ f)(v)=\overline{c o}(\partial g(f(v)) \partial f(v))$ where $\overline{c o}$ denotes the closed convex hull of a set. But $\partial g(f(v)) \partial f(v)$, which is the image by the continuous map $(P, Q) \mapsto P Q$ of the product of two compact sets, is compact, and the convex hull of a compact subset of $\mathbb{R}^{n}$, which is compact, coincides with its closed convex hull (see for instance [AB99, Corollary 5.18]), therefore $\overline{\mathrm{co}}(\partial g(f(v)) \partial f(v))=\operatorname{co}(\partial g(f(v)) \partial f(v))$, which shows (12), when $\ell=1$. The proof for $\ell>1$ follows readily from the result when $\ell=1$, together with the observation that the convex hull of a rectangular set is rectangular. 
We get as an easy corollary one inclusion in Theorem 4.1:

Corollary 4.4. If $f$ is as in Theorem 4.1, and if $k \geq 1$, then, $\partial f^{k}(v)=\operatorname{co}\left((\partial f(v))^{k}\right)$, for all eigenvectors $v$ of $f$. Moreover, $\mathscr{G}^{\mathrm{c}}\left(f^{k}\right) \supset \mathscr{G}^{\mathrm{c}}(f)^{k}$.

Proof. By (12), $\partial f^{k}(v)=\operatorname{co}(\partial f(v) \operatorname{co}(\cdots \operatorname{co} \partial f(v) \cdots))=\operatorname{co}(\partial f(v) \cdots \partial f(v))=$ co $\left((\partial f(v))^{k}\right)$. Assertion 1 of Corollary 2.6 shows that there is a matrix $P \in \partial f(v)$ such that $\mathscr{G}^{\mathrm{c}}(f)=\mathscr{G}^{\mathrm{f}}(P)$. Since $P^{k} \in(\partial f(v))^{k} \subset \partial f^{k}(v), \mathscr{G}^{\mathrm{f}}\left(P^{k}\right) \subset \mathscr{G}^{\mathrm{c}}\left(f^{k}\right)$, and, using Proposition 4.2, we get $\mathscr{G}^{\mathrm{c}}(f)^{k}=\mathscr{G}^{\mathrm{f}}(P)^{k}=\mathscr{G}^{\mathrm{f}}\left(P^{k}\right) \subset \mathscr{G}^{\mathrm{c}}\left(f^{k}\right)$.

To show the other inclusion, we shall need the following nonlinear version of Proposition 2.8, which relates final classes with invariant measures.

Proposition 4.5. Let $f$ denote a convex monotone homogeneous map $\mathbb{R}^{n} \rightarrow \mathbb{R}^{n}$ that has a fixed point, $v$, and let $F$ be a subset of $\{1, \ldots, n\}$. The following propositions are equivalent:

1. F is a union of elements of $\mathscr{C}^{\mathrm{c}}(f)$,

2. there exists a stochastic row vector $m$ with support $F$, such that $m f(x) \geq m x, \forall x \in \mathbb{R}^{n}$,

3. there exists a stochastic row vector $m$ with support $F$, such that $m f_{v}^{\prime}(x) \geq m x, \forall x \in \mathbb{R}^{n}$.

Proof. Without loss of generality, we assume that $v=0$ (otherwise, replace $f$ by $-v+f(\cdot+v)$ ). $(1 \Longrightarrow 2)$ Let $F$ be a union of elements of $\mathscr{C}^{\mathrm{c}}(f)=\mathscr{C}^{\mathrm{f}}(\partial f(0))$. Using the first assertion of Proposition 2.3, we can write $F$ as the union of disjoint sets $F_{1}, \ldots, F_{k} \in \mathscr{C}^{\mathrm{c}}(f)$. Moreover, there is a matrix $P \in \partial f(0)$ such that $F_{1}, \ldots, F_{k}$ are final classes of $P$. Indeed, pick $P^{(1)}, \ldots, P^{(k)} \in$ $\partial f(0)$ such that for all $1 \leq l \leq k, F_{l}$ is a final class of $P^{(l)}$. We denote by $P$ the matrix whose $i$-th row is equal to the $i$-th row of $P^{(l)}$, when $i \in F_{l}$, and to any element of $\partial f_{i}(0)$, when $i \notin F$. By rectangularity of $\partial f(0), P \in \partial f(0)$, and $F_{1}, \ldots, F_{k}$ are final classes of $P$.

Thus, $F$ is a union of final classes of $P$. It follows, by Proposition 2.8, that $P$ has an invariant measure $m$ with support $F$. Since $P \in \partial f(0)$, we get $f(x) \geq P x$ for all $x \in \mathbb{R}^{n}$, so $m f(x) \geq$ $m P x=m x$.

$(2 \Longrightarrow 3)$ Assume that there exists a stochastic row vector $m$ with support $F$, such that $m f(x) \geq$ $m x$ for all $x \in \mathbb{R}^{n}$. Then, $m \varepsilon^{-1} f(\varepsilon x) \geq m x$, for all $\varepsilon>0$. Taking the limit when $\varepsilon$ goes to zero, one gets $m f_{0}^{\prime}(x) \geq m x$ for all $x \in \mathbb{R}^{n}$.

$(3 \Longrightarrow 1)$ Assume now that there exists a stochastic row vector $m$ with support $F$, such that $m f_{0}^{\prime}(x) \geq m x$ for all $x \in \mathbb{R}^{n}$. Applying Property (10) to the coordinates of $f_{0}^{\prime}$ and using the rectangularity of $\partial f(0)$, we get

$$
m f_{0}^{\prime}(x)=m \sup _{P \in \partial f(0)} P x=\sup _{P \in \partial f(0)} m P x .
$$

Hence, setting $\mathscr{M}=\{m P, P \in \partial f(0)\}$, we see that the assumption on $m$ is equivalent to $\sup _{\nu \in \mathscr{M}} \nu x \geq m x$ for all $x \in \mathbb{R}^{n}$. In order to prove point 1 , it is sufficient to show that there exists $P \in \partial \vec{f}(0)$ such that $m P=m$, that is $m \in \mathscr{M}$. Indeed, by Proposition 2.8, this would imply that $F$ is a union of final classes of $P$, thus a union of elements of $\mathscr{C}^{\mathrm{c}}(f)$. Assume, by contradiction, 
that $m \notin \mathscr{M}$. Since $\partial f(0)$ is a convex compact subset of $\mathbb{R}^{n \times n}, \mathscr{M}$ is a convex compact subset of the space $\mathbb{R}^{1 \times n}$ of row vectors. Hence, the Hahn-Banach theorem implies that there exists a separating hyperplane, that is there exists a column vector $x \in \mathbb{R}^{n}$ and a real $\alpha$, such that $m x>\alpha$ and $\nu x<\alpha$ for all $\nu \in \mathscr{M}$. Then, $\sup _{\nu \in \mathscr{M}} \nu x \leq \alpha<m x$, a contradiction. We have proved that $m \in \mathscr{M}$, which shows point 1 .

We shall also need the following notion of additive recession function. Let $g: \mathbb{R}^{n} \rightarrow \mathbb{R}$ be an additively subhomogeneous map, that is a map such that $g(\lambda+x) \leq \lambda+g(x)$ for all $\lambda \geq 0$ and $x \in \mathbb{R}^{n}$. The sequence $-\rho+g(\rho+y)$ is nonincreasing with respect to $\rho>0$, allowing us to define the additive recession function $\tilde{g}: \mathbb{R}^{n} \rightarrow \mathbb{R} \cup\{-\infty\}$ of $g$ by

$$
\tilde{g}(y)=\lim _{\rho \rightarrow+\infty}-\rho+g(\rho+y) .
$$

If $g$ is monotone and convex, so does $\tilde{g}$. Moreover, the domain of $g^{*}$ is included in the set $\mathscr{S}_{n}^{-}=$ $\left\{p \in \mathbb{R}^{n} \mid \sum_{1 \leq i \leq n} p_{i} \leq 1, p_{1}, \ldots, p_{n} \geq 0\right\}$ of substochastic vectors, and $\tilde{g}$ is given by:

$$
\tilde{g}(y)=\sup _{q \in \operatorname{dom} g^{*} \cap \mathscr{S}_{n}} q \cdot y-g^{*}(q),
$$

where an empty supremum is equal to $-\infty$. By (15) and the nonexpansiveness of $g, \tilde{g}$ is either finite everywhere or identically equal to $-\infty$.

We define similarly the additive recession function of an additively subhomogeneous map $g$ : $\mathbb{R}^{n} \rightarrow \mathbb{R}^{n}$. If $g=\left(g_{1}, \ldots, g_{n}\right)$, we have $\tilde{g}=\left(\widetilde{g_{1}}, \ldots, \widetilde{g_{n}}\right)$. If $g$ is monotone and convex, and if $\tilde{g}$ has only finite entries, we have, by (15), $g(\rho+y)=\rho+\tilde{g}(y)+o(1)$, when $\rho$ goes to $+\infty$. So using the nonexpansiveness of $g$, we get $g \circ h(\rho+y)=g(\rho+\tilde{h}(y))+o(1)=\rho+\tilde{g} \circ \tilde{h}(y)+o(1)$, for any other map $h$ with the same properties as $g$, which leads to the following chain rule:

Lemma 4.6. Let $g$ and $h: \mathbb{R}^{n} \rightarrow \mathbb{R}^{n}$ be two monotone, convex and subhomogeneous maps, such that $\tilde{g}$ and $\tilde{h}$ take finite values. Then, $g \circ h: \mathbb{R}^{n} \rightarrow \mathbb{R}^{n}$ is monotone, convex, subhomogeneous, and $\widetilde{g \circ h}=\tilde{g} \circ \tilde{h}$.

For any $n \times n$ stochastic matrix $P$, we say that the set $F \subset\{1, \ldots, n\}$ is invariant by $P$ if $P_{i j}=0$ for all $i \in F$ and $j \notin F$ (that is, if it is invariant by the dynamics of the Markov chain of transition matrix $P$ ).

Lemma 4.7. Let $f$ denote a convex monotone homogeneous map $\mathbb{R}^{n} \rightarrow \mathbb{R}^{n}$ that has an eigenvector $v$. Then, there exists at least one critical class of $f$ that is invariant by all the matrices $P \in \partial f(v)$. We say that such a critical class is invariant by $f$.

Proof. Let $\mathscr{G}=\cup_{P \in \partial f(v)} \mathscr{G}(P)$ and $F$ denotes an arbitrary final class of $\mathscr{G}$. The set $F$ is clearly invariant by all the matrices $P \in \partial f(v)$. Since $\mathscr{G}$ is finite, there exists a finite set $\mathscr{P} \subset \partial f(v)$ such that $\mathscr{G}=\cup_{P \in \mathscr{P}} \mathscr{G}(P)$. Consider $\widehat{P}=|\mathscr{P}|^{-1}\left(\sum_{P \in \mathscr{P}} P\right)$. Using the convexity of $\partial f(v)$, we have $\widehat{P} \in \partial f(v)$. Since the graph of $\widehat{P}$ is equal to $\mathscr{G}, F$ is a final class of $\widehat{P}$, hence $F \in \mathscr{C}^{\mathrm{c}}(f)$. This is a critical class of $f$ since for all $P \in \partial f(v), P_{i j}=0$ for all $i \in F$ and $j \notin F$. 
Example 4.8. For the map $f: \mathbb{R}^{3} \rightarrow \mathbb{R}^{3}$,

$$
f(x)=\left(\begin{array}{c}
x_{2} \\
x_{2} \vee x_{3} \\
x_{3}
\end{array}\right)
$$

both $\{2\}$ and $\{3\}$ are critical classes, but only $\{3\}$ is invariant.

The last ingredient in the proof of Theorem 4.1 is a simple restriction operation. If $N$ is a subset of $\{1, \ldots, n\}$, we denote by $\mathrm{i}_{N}$ the trivial injection $\mathbb{R}^{N} \rightarrow \mathbb{R}^{n}$, which is defined by $\left(\mathrm{i}_{N}(x)\right)_{i}=x_{i}$ if $i \in N$, and $\left(\mathrm{i}_{N}(x)\right)_{i}=0$, otherwise. We define the restriction $f_{N N}: \mathbb{R}^{N} \rightarrow \mathbb{R}^{N}$ of $f$ to $N$ by $f_{N N}=\mathrm{r}_{N} \circ f \circ \mathrm{i}_{N}$. If $f$ is convex monotone and bihomogeneous, then $f_{N N}$ is clearly monotone, convex, multiplicatively homogeneous, and additively subhomogeneous. Indeed, $f(x)=f_{0}^{\prime}(x)=$ $\sup _{P \in \partial f(0)} P x$, so $f_{N N}(y)=\sup _{Q \in \partial f_{N N}(0)} Q y$, with $\partial f_{N N}(0)=\left\{P_{N N} \mid P \in \partial f(0)\right\}$. Recall that the critical graph of an additively subhomogeneous map has been defined in $\S 1.4$. We have the following lemma, which follows readily from the fact that for any $P \in \partial f(0), P_{F N}=0$, so that any final class of $P$ is included either in $N$ or in $F$.

Lemma 4.9. Let $F$ denote an invariant critical class of a convex monotone bihomogeneous map $f: \mathbb{R}^{n} \rightarrow \mathbb{R}^{n}$, and let $N=\{1, \ldots, n\} \backslash F$. Then, $f \circ \mathrm{i}_{N}=\mathrm{i}_{N} \circ f_{N N}, f_{F F} \circ \mathrm{r}_{F}=\mathrm{r}_{F} \circ f$ and the map $f_{F F}$ is additively homogeneous. Moreover, $\mathscr{G}^{\mathrm{c}}(f)=\mathscr{G}^{\mathrm{c}}\left(f_{N N}\right) \cup \mathscr{G}^{\mathrm{c}}\left(f_{F F}\right)$, and, when all the entries of $\widetilde{f_{N N}}$ are finite,

$$
\mathscr{G}^{\mathrm{c}}(f)=\mathscr{G}^{\mathrm{c}}\left(\widetilde{f_{N N}}\right) \cup \mathscr{G}^{\mathrm{c}}\left(f_{F F}\right)
$$

(the unions are disjoint).

In general, $f_{N N}$ is only additively subhomogeneous, since the $N \times N$ submatrices $P_{N N}$ are only substochastic.

Example 4.10. For the map $f$ of Example 4.8, we have $F=\{3\}, N=\{1,2\}$, and

$$
f_{N N}\left(\begin{array}{c}
x_{1} \\
x_{2}
\end{array}\right)=\left(\begin{array}{c}
x_{2} \\
x_{2} \vee 0
\end{array}\right), \widetilde{f_{N N}}\left(\begin{array}{c}
x_{1} \\
x_{2}
\end{array}\right)=\left(\begin{array}{c}
x_{2} \\
x_{2}
\end{array}\right), \quad f_{F F}\left(x_{3}\right)=x_{3}
$$

and $\mathscr{G}^{\mathrm{c}}(f)=\mathscr{G}^{\mathrm{c}}\left(\tilde{f}_{N N}\right) \cup \mathscr{G}^{\mathrm{c}}\left(f_{F F}\right)$ is the disjoint union of the two graphs consisting of a single loop at node 2 , and 3 , respectively.

Proof of Theorem 4.1. Without loss of generality, we assume that 0 is a fixed point of $f$. By definition of $\mathscr{G}^{\mathrm{c}}(f), \mathscr{G}^{\mathrm{c}}(f)=\mathscr{G}^{\mathrm{c}}\left(f_{0}^{\prime}\right)$. Moreover, Lemma 4.3 shows that $\left(f^{k}\right)_{0}^{\prime}=\left(f_{0}^{\prime}\right)^{k}$ so that $\mathscr{G}^{\mathrm{c}}\left(f^{k}\right)=\mathscr{G}^{\mathrm{c}}\left(\left(f^{k}\right)_{0}^{\prime}\right)=\mathscr{G}^{\mathrm{c}}\left(\left(f_{0}^{\prime}\right)^{k}\right)$. Thus, it is enough to prove Theorem 4.1 for maps of the form $f_{0}^{\prime}$, that is for convex monotone homogeneous maps that are also multiplicatively homogeneous.

We next reduce to the case where $\mathrm{N}^{\mathrm{c}}\left(f^{k}\right)=\{1, \ldots, n\}$. Indeed, let $N=\{1, \ldots, n\} \backslash \mathrm{N}^{\mathrm{c}}\left(f^{k}\right)$ and consider $g: \mathbb{R}^{n} \rightarrow \mathbb{R}^{n}$ defined by $g_{i}(x)=f_{i}(x) \vee x_{i}$ if $i \in N$ and $g_{i}(x)=f_{i}(x)$ otherwise. Using the inclusion already proved in Corollary 4.4, we get $\mathrm{N}^{\mathrm{c}}(f) \subset \mathrm{N}^{\mathrm{c}}\left(f^{k}\right)=\{1, \ldots, n\} \backslash N$, hence $\mathscr{G}^{\mathrm{c}}(g)$ is the disjoint union of $\mathscr{G}^{\mathrm{c}}(f)$ and of the set $\mathscr{L}$ of loops $i \rightarrow i$ with $i \in N$. This implies in particular that $\mathscr{G}^{\mathrm{c}}(g)^{k}$ is the disjoint union of $\mathscr{G}^{\mathrm{c}}(f)^{k}$ and $\mathscr{L}$, and that $\mathrm{N}^{\mathrm{c}}(g)=\mathrm{N}^{\mathrm{c}}(f) \cup N$. Since 
$g(0)=0$ and $g^{k} \geq f^{k}$, we get $\mathrm{N}^{\mathrm{c}}\left(g^{k}\right) \supset \mathrm{N}^{\mathrm{c}}\left(f^{k}\right)$ and $\mathscr{G}^{\mathrm{c}}\left(g^{k}\right) \supset \mathscr{G}^{\mathrm{c}}\left(f^{k}\right)$. Again by Corollary 4.4, we get $\mathrm{N}^{\mathrm{c}}\left(g^{k}\right) \supset \mathrm{N}^{\mathrm{c}}(g) \supset N$, hence, $\mathrm{N}^{\mathrm{c}}\left(g^{k}\right)=\{1, \ldots, n\}$. Now if $\mathscr{G}^{\mathrm{c}}\left(g^{k}\right) \subset \mathscr{G}^{\mathrm{c}}(g)^{k}$, then $\mathscr{G}^{\mathrm{c}}\left(f^{k}\right) \subset$ $\mathscr{G}^{\mathrm{c}}\left(g^{k}\right) \subset \mathscr{G}^{\mathrm{c}}(g)^{k}=\mathscr{G}^{\mathrm{c}}(f)^{k} \cup \mathscr{L}$, and since $\mathrm{N}^{\mathrm{c}}\left(f^{k}\right)=\{1, \ldots, n\} \backslash N$, we have $\mathscr{G}^{\mathrm{c}}\left(f^{k}\right) \subset \mathscr{G}^{\mathrm{c}}(f)^{k}$, which, together with the inclusion proved in Corollary 4.4, yields $\mathscr{G}^{\mathrm{c}}\left(f^{k}\right)=\mathscr{G}^{\mathrm{c}}(f)^{k}$.

We are now reduced to show the following proposition, $\left(\mathrm{P}_{n}\right)$ : for all convex monotone bihomogeneous maps $f: \mathbb{R}^{n} \rightarrow \mathbb{R}^{n}$, and for all $k \geq 1$, such that $\mathrm{N}^{\mathrm{c}}\left(f^{k}\right)=\{1, \ldots, n\}$, we have $\mathscr{G}^{\mathrm{c}}\left(f^{k}\right) \subset \mathscr{G}^{\mathrm{c}}(f)^{k}$. We shall prove $\left(\mathrm{P}_{n}\right)$ by induction on the dimension $n$. Proposition $\left(\mathrm{P}_{1}\right)$ trivially holds, since in dimension 1 , the only possible map $f$ is the identity map. Assume now that $\left(\mathrm{P}_{\ell}\right)$ holds for all dimensions $\ell<n$, and consider a map $f: \mathbb{R}^{n} \rightarrow \mathbb{R}^{n}$ as in $\left(\mathrm{P}_{n}\right)$.

Let $F$ be an invariant critical class of $f$ (as in Lemma 4.7), let $N=\{1, \ldots, n\} \backslash F$, and put $g=f_{N N}$ and $h=f_{F F}$. Assume first that all the entries of $\widetilde{g}$ are finite. Then, by (17), $\mathscr{G}^{\mathrm{c}}(f)=$ $\mathscr{G}^{\mathrm{c}}(\widetilde{g}) \cup \mathscr{G}^{\mathrm{c}}(h)$. Since $F$ is also invariant for $f^{k}$, we also have $\mathscr{G}^{\mathrm{c}}\left(f^{k}\right)=\mathscr{G}^{\mathrm{c}}\left(\left(\widetilde{\left.f^{k}\right)_{N N}}\right) \cup \mathscr{G}^{\mathrm{c}}\left(\left(f^{k}\right)_{F F}\right)\right.$. Using the first part of Lemma 4.9, we obtain $\left(f^{k}\right)_{N N}=\mathrm{r}_{N} \circ f^{k} \circ i_{N}=g^{k}$ and $\left(f^{k}\right)_{F F}=h^{k}$. Moreover, if the entries of $\widetilde{g}$ are finite, Lemma 4.6 yields $\widetilde{g^{k}}=\widetilde{g}^{k}$, so $\mathscr{G}^{\mathrm{c}}\left(f^{k}\right)=\mathscr{G}^{\mathrm{c}}\left(\widetilde{g}^{k}\right) \cup \mathscr{G}^{\mathrm{c}}\left(h^{k}\right)$. Thus, in order to prove $\mathscr{G}^{c}\left(f^{k}\right) \subset \mathscr{G}^{c}(f)^{k}$, it is sufficient to prove the same inclusion for $h$ and $\widetilde{g}$ and to prove that $\widetilde{g}$ has finite entries.

Let us first prove $\mathscr{G}^{\mathrm{c}}\left(h^{k}\right) \subset \mathscr{G}^{\mathrm{c}}(h)^{k}$. Since $F$ is an invariant critical class of $f, \mathscr{G}^{\mathrm{c}}(h)$ is strongly connected and contains all the nodes of $F$. By Assertion 1 of Corollary 2.6, there exists $P \in \partial h(0)$ such that $\mathscr{G}^{\mathrm{c}}(h)=\mathscr{G}^{\mathrm{f}}(P)$, hence $P$ is irreducible and $\mathscr{G}^{\mathrm{c}}(h)=\mathscr{G}(P)$. Moreover, for all $Q \in \partial h(0)$, the matrix $\frac{Q+P}{2}$ belongs to $\partial h(0)$ and is irreducible, so $\mathscr{G} f(Q) \subset \mathscr{G}(Q) \subset \mathscr{G}\left(\frac{Q+P}{2}\right)=$ $\mathscr{G}^{\mathrm{f}}\left(\frac{Q+P}{2}\right) \subset \mathscr{G}^{\mathrm{c}}(h)$. Defining $\mathscr{G}(\mathscr{P})=\cup_{P \in \mathscr{P}} \mathscr{G}(P)$ for all sets $\mathscr{P}$ of stochastic matrices, we get $\mathscr{G}^{2}(h)=\mathscr{G}(\partial h(0))$. Hence, $\mathscr{G}^{\mathrm{c}}\left(h^{k}\right) \subset \mathscr{G}\left(\partial\left(h^{k}\right)(0)\right)=\mathscr{G}\left(\operatorname{co}\left((\partial h(0))^{k}\right)\right)=\mathscr{G}\left((\partial h(0))^{k}\right) \subset$ $\mathscr{G}(\partial h(0))^{k}=\mathscr{G}^{\mathrm{c}}(h)^{k}$.

Let us now prove that all the entries of $\widetilde{g}$ are finite. This is indeed equivalent to the property $g(1)=1$, where 1 denotes the unit vector (which means that $\partial g(0) \cap \mathscr{S}_{N N} \neq \varnothing$ ). We know that $g(1) \leq 1$, so by monotonicity of $g, g^{k}(1) \leq \cdots \leq g(1) \leq 1$. On the other hand, by Proposition 4.5, there exists a stochastic (row) vector $m$ with positive entries (i.e., the support of $m$ is $\{1, \ldots n\}$ ), such that $m f^{k}(x) \geq m x$ for all $x \in \mathbb{R}^{n}$. By Lemma 4.9, $f \circ \mathrm{i}_{N}=\mathrm{i}_{N} \circ g$, hence $f^{k} \circ \mathrm{i}_{N}=\mathrm{i}_{N} \circ g^{k}$. Applying $m f^{k}(x) \geq m x$ to $x=\mathrm{i}_{N}(y)$, we obtain $m_{N} g^{k}(y) \geq m_{N} y$, where $m_{N}$ is the restriction of $m$ to the set $N\left(m_{N}\right.$ has positive entries since all the entries of $m$ are positive). So $m_{N} 1 \geq$ $m_{N} g(1) \geq \cdots \geq m_{N} g^{k}(1) \geq m_{N} 1$. This yields $m_{N} 1=m_{N} g(1)$. Using the positivity of $m_{N}$ and the inequality $g(1) \leq 1$, we get $1=g(1)$.

We finally show that $\mathscr{G}^{c}\left(\widetilde{g}^{k}\right) \subset \mathscr{G}^{\mathrm{c}}(\widetilde{g})^{k}$, by using the induction assumption. Indeed, since $F \neq \varnothing$, the cardinality of $N$, which is the dimension of the space on which $g$ operates, is strictly less than $n$. Moreover, since $m_{N} g^{k}(y) \geq m_{N} y$ for all $y \in \mathbb{R}^{N}$, we deduce that $m_{N} \widetilde{g^{k}}(y) \geq m_{N} y$. Since $\widetilde{g}$ has finite entries, $\widetilde{g^{k}}=\widetilde{g}^{k}$, so that $m_{N} \widetilde{g}^{k}(y) \geq m_{N} y$ for all $y \in \mathbb{R}^{N}$, and Proposition 4.5 shows that $\mathrm{N}^{\mathrm{c}}\left(\widetilde{g}^{k}\right)=N$. The induction assumption can then be applied to $\widetilde{g}$, and yields $\mathscr{G}^{\mathrm{c}}\left(\widetilde{g}^{k}\right) \subset \mathscr{G}^{\mathrm{c}}(\widetilde{g})^{k}$. This concludes the proof of $\left(\mathrm{P}_{n}\right)$. 


\section{Cyclicity Theorem for Convex Monotone Homogeneous Maps}

In this section, we use our knowledge of the eigenspace of $f$ to study the asymptotic behavior of $f^{k}$ when $k$ tends to $+\infty$. In particular, we are interested in the periodic orbits of $f$, which are of the form $\left\{f^{k}(x)\right\}_{k \in \mathbb{N}}$, with $f^{c}(x)=x$ for some $c \geq 1$. The set of such $c$ is exactly the set of multiples of a positive integer, which is the length of the orbit $\left\{f^{k}(x)\right\}_{k \in \mathbb{N}}$.

Let us first recall some more or less classical facts on periodic orbits of stochastic matrices. The cyclicity $\mathrm{c}(\mathscr{G})$ of a strongly connected graph $\mathscr{G}$ is the gcd of the lengths of the circuits of $\mathscr{G}$. The cyclicity of a graph $\mathscr{G}$ with strongly connected component $\mathscr{G}_{1}, \ldots, \mathscr{G}_{s}$ is defined by c $(\mathscr{G})=$ $\operatorname{lcm}\left(\mathrm{c}\left(\mathscr{G}_{1}\right), \ldots, \mathrm{c}\left(\mathscr{G}_{s}\right)\right)$. For a stochastic matrix $P$, we define the cyclicity of $P$ as: $\mathrm{c}(P)=\mathrm{c}\left(\mathscr{G}^{\mathrm{f}}(P)\right)$. The name "cyclicity" is justified by the following result.

Proposition 5.1. If $P$ is a stochastic matrix, then, the length of any periodic orbit of $x \mapsto P x$ divides $\mathrm{c}(P)$.

Proof. If $P$ is a stochastic matrix with cyclicity $c=\mathrm{c}(P)$, then the Perron-Frobenius theorem shows that $P^{\ell c} \rightarrow \Pi$ when $\ell \rightarrow \infty$, where $\Pi$ is the spectral projector of $P^{c}$ for the eigenvalue 1 . If $P^{k} x=x$, then, $x=\lim _{\ell \rightarrow \infty} P^{\mathrm{lcm}(k, c) \ell} x=\Pi x$. Since the spectral projector satisfies $P^{c} \Pi=\Pi$, we get $x=P^{c} x$, which shows that the length of any periodic orbit of $P$ divides $c$.

More generally, we define the cyclicity $\mathrm{c}(f)$ of a convex monotone homogeneous map $f$ as the cyclicity of its critical graph $\mathrm{c}\left(\mathscr{G}^{\mathrm{c}}(f)\right)$.

Proposition 5.2. Let $f$ denote a convex monotone homogeneous map that has an eigenvector $v$. Then,

$$
\mathrm{c}(f)=\operatorname{gcd}\left\{\mathrm{c}(P) \mid P \in \partial f(v), \mathrm{N}^{\mathrm{f}}(P)=\mathrm{N}^{\mathrm{c}}(f)\right\} \text {. }
$$

Moreover, there exists $P \in \partial f(v)$ such that $\mathrm{N}^{\mathrm{f}}(P)=\mathrm{N}^{\mathrm{c}}(f)$ and $\mathrm{c}(P)=\mathrm{c}(f)$.

Proof. Let $P \in \partial f(v)$ such that $\mathrm{N}^{\mathrm{f}}(P)=\mathrm{N}^{\mathrm{c}}(f)$. Since $\mathscr{G}^{\mathrm{f}}(P) \subset \mathscr{G}^{\mathrm{c}}(f)$, and since both graphs have the same set of nodes, each critical class $C$ of $f$ can be partitioned into final classes of $P$, $C=F_{1} \cup \ldots \cup F_{r}$, and $\left.\left.\mathscr{G}^{\mathrm{f}}(P)\right|_{C} \subset \mathscr{G}^{\mathrm{c}}(f)\right|_{C}$, where $\left.\mathscr{G}\right|_{C}$ denotes the restriction of the graph $\mathscr{G}$ to $C$. Hence, the cyclicity of $\left.\mathscr{G}^{\mathrm{c}}(f)\right|_{C}$, which is equal to the gcd of the lengths of circuits of $\left.\mathscr{G}^{\mathrm{c}}(f)\right|_{C}$ divides $\mathrm{c}\left(\left.\mathscr{G}^{\mathrm{f}}(P)\right|_{C}\right)=\operatorname{lcm}\left(\mathrm{c}\left(P_{F_{1} F_{1}}\right), \ldots, \mathrm{c}\left(P_{F_{r}, F_{r}}\right)\right)$. Since this divisibility holds for all critical classes $C$ of $f, \mathrm{c}(f)$ divides $\mathrm{c}(P)$. Conversely, Assertion 1 of Corollary 2.6, there is a matrix $P \in \partial f(v)$ such that $\mathscr{G}^{\mathrm{f}}(P)=\mathscr{G}^{\mathrm{c}}(f)$, which shows that the right hand side of (18) divides c $(f)$.

Proposition 5.3. Let $f$ be a convex monotone homogeneous map that has a fixed point and let $\mathscr{G}_{1}, \ldots, \mathscr{G}_{s}$ be the strongly connected components of $\mathscr{G}^{\mathrm{c}}(f)$. Then, $f^{\mathrm{c}(f)}$ has $\mathrm{c}\left(\mathscr{G}_{1}\right)+\cdots+\mathrm{c}\left(\mathscr{G}_{s}\right)$ critical classes and has cyclicity 1.

Proof. From Theorem 4.1, we get $\mathscr{G}^{\mathrm{c}}\left(f^{\mathrm{c}(f)}\right)=\mathscr{G}^{\mathrm{c}}(f)^{\mathrm{c}(f)}=\cup_{i=1}^{s} \mathscr{G}_{i}^{\mathrm{c}(f)}$ (disjoint union). For any strongly connected graph $\mathscr{G}$ with cyclicity $c$ and any integer $k, \mathscr{G}^{k c}$ has $c$ strongly connected components with cyclicity 1 (this is a well known result which follows from the decomposition of $\mathscr{G}$ into cyclic classes, see e.g. [KSK66, Chapter 6, §3] and [BP94, Chapter 2, §2]). Since c $\left(\mathscr{G}_{i}\right)$ divides $\mathrm{c}(f)$, we obtain that $\mathscr{G}^{\mathrm{c}}\left(f^{\mathrm{c}(f)}\right)$ has $\mathrm{c}\left(\mathscr{G}_{1}\right)+\cdots+\mathrm{c}\left(\mathscr{G}_{s}\right)$ strongly connected components with cyclicity 1 . 
We shall also need the following classical fact.

Proposition 5.4. A monotone homogeneous map $\mathbb{R}^{n} \rightarrow \mathbb{R}^{n}$ which has a periodic orbit has a fixed point.

Proof. If $f$ has a periodic orbit, there is an $x \in \mathbb{R}^{n}$ and $k \geq 1$ such that $f^{k}(x)=x$. Consider $z=x \wedge f(x) \wedge \ldots \wedge f^{k-1}(x)$, By monotonicity of $f, f(z) \leq f(x) \wedge f^{2}(x) \wedge \ldots \wedge f^{k}(x)=z$. Thus, the sequence $\left\{f^{\ell}(z)\right\}_{\ell \in \mathbb{N}}$ is nonincreasing, and since by nonexpansiveness of $f,\left|f^{\ell}(z)-f^{\ell}(x)\right| \leq$ $|z-x|$ is bounded, $f^{\ell}(z)$ which is bounded and nonincreasing converges to a limit which is an eigenvector of $f$.

Theorem 5.5. The length of any periodic orbit of a convex monotone homogeneous map $f$ divides $c(f)$.

Proof. If $f$ has a periodic orbit $\left\{f^{k}(x)\right\}_{k \in \mathbb{N}}$, then, by Proposition 5.4, $f$ has a fixed point $v$. It suffices to prove Theorem 5.5 when $c(f)=1$. Indeed, if $\mathrm{c}(f) \neq 1$, consider $g=f^{c(f)}$, which is such that $\mathrm{c}(g)=1$ (by Proposition 5.3) and $\left\{g^{k}(x)\right\}_{k \in \mathbb{N}}$ is periodic. If we know that Theorem 5.5 holds for $g$, we get $x=g(x)=f^{c(f)}(x)$, hence the length of the periodic orbit $\left\{f^{k}(x)\right\}_{k \in \mathbb{N}}$ divides $c(f)$, which yields Theorem 5.5 in the general case.

Let us now prove the theorem when $\mathrm{c}(f)=1$. Using Proposition 5.2, we can find a matrix $P \in \partial f(v)$ such that $\mathrm{c}(P)=\mathrm{c}(f)=1$ and $\mathrm{N}^{\mathrm{f}}(P)=\mathrm{N}^{\mathrm{c}}(f)$. Using $f(v)=v$ and $f(y)-f(v) \geq$ $P(y-v)$, we get after an immediate induction,

$$
f^{k}(x) \geq P^{k}(x-v)+v \quad \forall k \in \mathbb{N} .
$$

In particular, if $c$ is the length of the orbit $\left\{f^{k}(x)\right\}_{k \in \mathbb{N}}, x=f^{c}(x) \geq P^{c}(x-v)+v$.

To make the proof more intuitive, we shall first show the theorem when $\mathrm{N}^{\mathrm{c}}(f)=\{1, \ldots, n\}$. By Proposition 4.2, $P^{c}$ again has $\{1, \ldots, n\}$ as union of final classes, and applying Lemma 2.9 to $x-v \geq P^{c}(x-v)$, we get $x-v=P^{c}(x-v)$. Since $P$ has cyclicity 1 , this implies that $P(x-v)=$ $x-v$, and using (19), we get $f(x) \geq x$. By monotonicity of $f$, we get $x=f^{c}(x) \geq \cdots \geq f(x) \geq x$. Thus, $f(x)=x$, and $c=1=\mathrm{c}(f)$.

Consider now the general case where $F=\mathrm{N}^{\mathrm{c}}(f) \neq\{1, \ldots, n\}$. As before, we get $x-v \geq$ $P^{c}(x-v)$. By Lemma 2.9 and Proposition 4.2, this yields $x-v=P^{c}(x-v)$ on $\mathrm{N}^{\mathrm{f}}\left(P^{c}\right)=$ $\mathrm{N}^{\mathrm{f}}(P)=F$. Since $P_{F N}=0$ for $N=\{1, \ldots, n\} \backslash F$, this equation can be rewritten as $(x-v)_{F}=$ $\left(P_{F F}\right)^{c}(x-v)_{F}$. Since $c\left(P_{F F}\right)=c(P)=c(f)=1$, we deduce $(x-v)_{F}=P_{F F}(x-v)_{F}$. Then, for all $k \geq 1,\left(P_{F F}\right)^{k}(x-v)_{F}=(x-v)_{F}$ or equivalently $\left(P^{k}(x-v)\right)_{F}=(x-v)_{F}$. Putting this in (19), we get $f^{k}(x) \geq x$ on $F$. Consider now $z=x \wedge f(x) \wedge \cdots \wedge f^{c-1}(x)$, which coincides with $x$ on $F$. By monotonicity of $f, f(z) \leq z$. Hence, by Lemma 3.3, $w=f^{\omega}(z)$ is a fixed point of $f$ and it coincides with $z$ on $\mathrm{N}^{\mathrm{c}}(f)=F$. Since $f(w)=w$, we get $f^{c}(w)=w$. So $w$ and $x$ are two fixed points of $f^{c}$, which coincide on $F=\mathrm{N}^{\mathrm{c}}(f)=\mathrm{N}^{\mathrm{c}}\left(f^{c}\right)$. This implies that $w=x$ by Theorem 3.4. Hence, $f(x)=x$, and by the minimality of $c$ (as the length of the orbit of $x$ ), we get $c=1$.

Corollary 5.6. The lengths of periodic orbits of convex monotone homogeneous maps $\mathbb{R}^{n} \rightarrow \mathbb{R}^{n}$ are exactly the orders of the elements of the symmetric group on $n$ letters. 
Combining Theorem 5.5 with the theorem of Nussbaum [Nus90] and Sine [Sin90], we get:

Corollary 5.7. If $f$ is a convex monotone homogeneous map $\mathbb{R}^{n} \rightarrow \mathbb{R}^{n}$ with eigenvalue $\lambda$, then, for all $x \in \mathbb{R}^{n}, f^{k \mathrm{c}(f)}(x)-k \mathrm{c}(f) \lambda$ converges when $k \rightarrow \infty$.

In other words, introducing the normalized map $g=f-\lambda$, which is such that $g^{k}=f^{k}-k \lambda$, we see that any orbit $\left\{g^{k}(x)\right\}_{k \in \mathbb{N}}$ of $g$ converges to a periodic orbit of length at most $c(g)=c(f)$. We also get, as an immediate consequence of Theorem 5.5:

Corollary 5.8. For any convex monotone homogeneous map $f$ that has a fixed point, the union of the periodic orbits of $f$ is exactly the set $\mathscr{E}\left(f^{\mathrm{c}(f)}\right)$ of fixed points of $f^{\mathrm{c}(f)}$.

Combining Corollary 5.8, Proposition 5.3, Theorem 4.1 and Theorem 3.4, we obtain:

Corollary 5.9. Let $f, \mathscr{G}_{1}, \ldots, \mathscr{G}_{s}$ be as in Proposition 5.3, and let $C=\mathrm{N}^{\mathrm{c}}(f)$. Then, $\mathrm{r}_{C}$ sends bijectively the union of periodic orbits, $\mathscr{E}\left(f^{\mathrm{c}(f)}\right)$, to a convex inf-subsemilattice of $\mathbb{R}^{C}$, whose dimension is at most equal to $\mathrm{m}\left(f^{\mathrm{c}(f)}\right)=\mathrm{c}\left(\mathscr{G}_{1}\right)+\cdots+\mathrm{c}\left(\mathscr{G}_{s}\right)$.

\section{Piecewise Affi ne Convex Monotone Homogeneous Maps}

\subsection{Dimension of the eigenspace}

As discussed in $\$ 1.2$, the dimension of the eigenspace of a piecewise affine convex monotone homogeneous map was characterized by Romanovsky [Rom73] and by Schweitzer and Federgruen [SF78]: this shows that the bound on the dimension given in Corollary 3.6, is attained when $f$ is piecewise affine. In this subsection, we give an independent proof of this fact, which shows some qualitative properties of $\mathscr{E}(f)$ (connection between $\mathscr{E}(f)$ and $\mathscr{E}\left(f_{v}^{\prime}\right)$, for any eigenvector $v$, role of invariant critical classes).

Let us first recall some basic definitions and facts. A polyhedron is an intersection of finitely many half-spaces. A map $f: \mathbb{R}^{n} \rightarrow \mathbb{R}$ is piecewise affine if $\mathbb{R}^{n}$ can be covered by finitely many polyhedra such that the restriction of $f$ to each polyhedron is affine. The following result is an immediate consequence of classical results on convex maps with polyhedral epigraphs [Roc70].

Proposition 6.1. A convex map $f: \mathbb{R}^{n} \rightarrow \mathbb{R}$ is piecewise affine if, and only if, there exists a finite set $\mathscr{P} \subset \operatorname{dom} f^{*}$ such that

$$
f(x)=\max _{p \in \mathscr{P}}\left(p \cdot x-f^{*}(p)\right) .
$$

It will be useful to consider bihomogeneous maps.

Corollary 6.2. A piecewise affine convex monotone bihomogeneous map $f: \mathbb{R}^{n} \rightarrow \mathbb{R}$ can be written as

$$
f(x)=\max _{p \in \mathscr{P}} p \cdot x
$$

where $\mathscr{P}$ is a finite set of stochastic vectors. 
Proof. By Proposition 2.1, $\operatorname{dom} f^{*} \subset \mathscr{S}_{n}$, therefore, the finite set $\mathscr{P}$ in (20) is composed of stochastic vectors, and since $f^{*}$ takes only the values 0 and $+\infty$ when $f$ is multiplicatively homogeneous, we get (21).

The following simple fact shows that eigenspaces of convex piecewise affine maps can be effectively computed.

Corollary 6.3. The eigenspace of a convex piecewise affine monotone homogeneous map $f: \mathbb{R}^{n} \rightarrow$ $\mathbb{R}^{n}$ is a finite union of polyhedra.

Proof. We assume, without loss of generality, that $f$ has eigenvalue 0. By Propositions 2.1 and 6.1, we can write each coordinate of $f$ as $f_{i}(x)=\max _{p \in \mathscr{P}_{i}}\left(p \cdot x-f_{i}^{*}(p)\right)$, where $\mathscr{P}_{i}$ is a finite subset of $\mathscr{S}_{n}$. To any $i \in\{1, \ldots, n\}$ and $p \in \mathscr{P}_{i}$, we associate the (possibly empty) polyhedron $K_{i, p}=\left\{x \in \mathbb{R}^{n} \mid x_{i}=p \cdot x-f_{i}^{*}(p) \geq q \cdot x-f_{i}^{*}(q), \forall q \in \mathscr{P}_{i}\right\}$. We denote by $\Phi$ the set of maps $\varphi:\{1, \ldots, n\} \rightarrow \mathscr{P}_{1} \cup \ldots \cup \mathscr{P}_{n}$ such that $\varphi(i) \in \mathscr{P}_{i}$, for all $i \in\{1, \ldots, n\}$. To $\varphi \in \Phi$, we associate the polyhedron $K_{\varphi}=\cap_{1 \leq i \leq n} K_{i, \varphi(i)}$. If $x=f(x)$, then, for each $i$, by finiteness of $\mathscr{P}_{i}$, we have $x_{i}=\max _{p \in \mathscr{P}_{i}} p \cdot x-f_{i}^{*}(p)=\varphi(i) \cdot x-f_{i}^{*}(\varphi(i))$, for some $\varphi(i) \in \mathscr{P}_{i}$. This shows that $\mathscr{E}(f) \subset \cup_{\varphi \in \Phi} K_{\varphi}$. Since the other inclusion holds trivially, $\mathscr{E}(f)=\cup_{\varphi \in \Phi} K_{\varphi}$ is a finite union of polyhedra.

The proof of Corollary 6.3 yields an algorithm (with exponential execution time) to compute $\mathscr{E}(f)$, but it does not tell much about the geometry of $\mathscr{E}(f)$. We shall next prove more qualitative properties.

The following observation shows that the directional derivative $f_{v}^{\prime}$ defined in Section 4 is a "tangent" map of $f$.

Lemma 6.4. If $f: \mathbb{R}^{n} \rightarrow \mathbb{R}$ is a piecewise affine convex map, then, for all $v \in \mathbb{R}^{n}$, there is a neighborhood $V$ of $v$ such that

$$
f(x)=f(v)+f_{v}^{\prime}(x-v) \quad \forall x \in V .
$$

Proof. The definition of $\partial f(v)$ together with (10) show that the inequality $\geq$ holds in (22). To show the other inequality, we write $f$ as (20), and set $\mathscr{Q}=\mathscr{P} \cap \partial f(v), \mathscr{R}=\mathscr{P} \backslash \mathscr{Q}$. We can write $f=g \vee h$ where

$$
g(x)=\max _{p \in \mathscr{P} \cap \partial f(v)}\left(p \cdot x-f^{*}(p)\right)=f(v)+\max _{p \in \mathscr{Q}} p \cdot(x-v)
$$

(since $f(v)=p \cdot v-f^{*}(p)$, for all $p \in \partial f(v)$ ), and $h(x)=\max _{p \in \mathscr{R}}\left(p \cdot x-f^{*}(p)\right.$ ). The functions $g$ and $h$ are continuous and satisfy $f(v)=g(v)>h(v)=\max _{p \in \mathscr{P} \backslash \partial f(v)}\left(p \cdot v-f^{*}(v)\right)$, where the strict inequality follows from the characterization (2) of $\partial f(v)$ and from the finiteness of $\mathscr{P}$. Therefore, there is a neighborhood $V$ of $v$ such that $f(x)=g(x) \vee h(x)=g(x)$ for all $x \in V$. Combining this with (23) and (10), we get $f(x)=g(x) \leq f(v)+f_{v}^{\prime}(x-v)$ for all $x \in V$.

Our interest in $f_{v}^{\prime}$ stems from the following reduction. 
Lemma 6.5. Let $f$ be a piecewise affine convex monotone homogeneous map $\mathbb{R}^{n} \rightarrow \mathbb{R}^{n}$ with eigenvector $v$. Then, the dimensions of $\mathscr{E}(f)$ and $\mathscr{E}\left(f_{v}^{\prime}\right)$ are the same.

Proof. We first note that by construction of $f_{v}^{\prime}$, the critical classes for $f$ and $f_{v}^{\prime}$ are the same. Let $C=\mathrm{N}^{\mathrm{c}}(f)=\mathrm{N}^{\mathrm{c}}\left(f_{v}^{\prime}\right)$. By Corollary 3.6, there is a set $U \subset \mathscr{E}\left(f_{v}^{\prime}\right)$ such that $\mathrm{r}_{C}(U)$ is a convex set of $\operatorname{dimension} \operatorname{dim} \mathscr{E}\left(f_{v}^{\prime}\right)$. Of course, we may choose a bounded $U$. Then, picking $V$ as in Lemma 6.4, we get $v+\epsilon U \subset V$, for $\epsilon$ small enough, and applying (22), we get

$$
f(v+\epsilon u)=f(v)+f_{v}^{\prime}(\epsilon u)=\lambda+v+\epsilon u \quad \forall u \in U,
$$

which shows that $v+\epsilon U \subset \mathscr{E}(f)$. Therefore, $\operatorname{dim} \mathscr{E}(f) \geq \operatorname{dim} \mathscr{E}\left(f_{v}^{\prime}\right)$. To show the other inequality, we take a set $U^{\prime} \subset \mathscr{E}(f)$ such that $\mathrm{r}_{C}\left(U^{\prime}\right)$ is a convex set of dimension $\operatorname{dim} \mathscr{E}(f)$. Then, by convexity of $\mathscr{E}^{\mathrm{c}}(f)$, for all $0<\epsilon<1, \mathrm{r}_{C}\left((1-\epsilon) v+\epsilon U^{\prime}\right) \subset \mathscr{E}^{\mathrm{c}}(f)$. We may choose a bounded $U^{\prime}$. Then, taking $V_{\epsilon}=\mathrm{r}_{C}^{-1}\left(\mathrm{r}_{C}\left((1-\epsilon) v+\epsilon U^{\prime}\right)\right) \subset \mathscr{E}(f)$ and using the continuity of $\mathrm{r}_{C}^{-1}$ from $\mathscr{E}^{\mathrm{c}}(f)$ to $\mathscr{E}(f)$ (which follows from the last assertion of Theorem 3.4), we get $V_{\epsilon} \subset V$ for $\epsilon$ small enough, and using (22) again, we get, for all $w \in V_{\epsilon}$,

$$
w-v=f(w)-\lambda-v=f(v)+f_{v}^{\prime}(w-v)-\lambda-v=f_{v}^{\prime}(w-v),
$$

which shows that $V_{\epsilon}-v \subset \mathscr{E}\left(f_{v}^{\prime}\right)$. Therefore, $\operatorname{dim} \mathscr{E}\left(f_{v}^{\prime}\right) \geq \operatorname{dim} \mathscr{E}(f)$.

We next give a new proof of the following result, equivalent forms of which were proved by Romanovsky, and Schweitzer and Federgruen.

Theorem 6.6 (Cf. [Rom73, Th. 3], [SF78, Th. 5.1 and Th. 5.3]). The dimension of the eigenspace of a piecewise affine convex monotone homogeneous map $f$ is equal to the number of critical classes, $\mathrm{m}(f)$.

We shall reduce to the special case of maps $g \geq I$ with eigenvalue 0 (where $I$ denotes the identity map). This case is simpler because, when $g \geq I$,

$$
\mathscr{E}(g)=\left\{x \in \mathbb{R}^{n} \mid g(x)=x\right\}=\left\{x \in \mathbb{R}^{n} \mid g(x) \leq x\right\}=\mathscr{E}^{+}(g)
$$

Lemma 6.7. If $g: \mathbb{R}^{n} \rightarrow \mathbb{R}^{n}$ is a piecewise affine convex monotone bihomogeneous map such that $g \geq I$, then, $\mathscr{E}(g)$ is a convex set of dimension $\mathrm{m}(g)$.

Before proving Lemma 6.7, let us show that it implies Theorem 6.6. Let $v$ denote any eigenvector of $f$, and consider the maps $f_{v}^{\prime}$ and $g=f_{v}^{\prime} \vee I$, which both are piecewise affine, convex, monotone and bihomogeneous. Let $s=\mathrm{m}\left(f_{v}^{\prime}\right)$, let $C_{1}, \ldots, C_{s}$ denote the critical classes of $f_{v}^{\prime}$ (which are also those of $f$ ), and $C=\mathrm{N}^{\mathrm{c}}\left(f_{v}^{\prime}\right)=C_{1} \cup \ldots \cup C_{s}$. It is immediate to check that $g$ also has $C_{1}, \ldots, C_{s}$ as critical classes, and that is has additional critical classes of the form $\{i\}$, where $i$ is any element of the complement $N=\{1, \ldots, n\} \backslash C$. Thus, $\mathrm{m}(g)=s+|N|$. Applying Lemma 6.7 to $g$, we get that $\operatorname{dim} \mathscr{E}(g)=s+|N|$. Let $S$ denote any section of $f_{v}^{\prime}$ (recall that sections were defined before Corollary 3.6). Then, $S^{\prime}=S \cup N$ is a section of $g$, and, by Corollary 3.6, $\mathrm{r}_{S^{\prime}}(\mathscr{E}(g))$ is a convex set of dimension $s+|N|=\left|S^{\prime}\right|$, which implies that $\mathbf{r}_{S}(\mathscr{E}(g))$ is a convex set of dimension $s$.

RR $n^{\circ} 4273$ 
Using Lemma 3.3, we see that $\left(f_{v}^{\prime}\right)^{\omega}$ sends $\mathscr{E}(g)=\mathscr{E}^{+}\left(f_{v}^{\prime}\right)$ to $\mathscr{E}\left(f_{v}^{\prime}\right)$, and that $\mathrm{r}_{S}(\mathscr{E}(g))$ coincides with $\mathrm{r}_{S}\left(\mathscr{E}\left(f_{v}^{\prime}\right)\right)(S \subset C)$. Therefore, $\mathrm{r}_{S}\left(\mathscr{E}\left(f_{v}^{\prime}\right)\right)$ has dimension $s$, which implies, by Corollary 3.6, that $\operatorname{dim} \mathscr{E}\left(f_{v}^{\prime}\right)=s=\mathrm{m}\left(f_{v}^{\prime}\right)$. By Lemma 6.5, we get $\operatorname{dim} \mathscr{E}(f)=\operatorname{dim} \mathscr{E}\left(f_{v}^{\prime}\right)=\mathrm{m}\left(f_{v}^{\prime}\right)=$ $\mathrm{m}(f)$, which shows Theorem 6.6.

Proof of Lemma 6.7. The convexity of $\mathscr{E}(g)$ follows from (24). We shall prove that $\operatorname{dim} \mathscr{E}(g)=$ $\mathrm{m}(g)$ by induction on the dimension $n$. (The constructions of the proof are illustrated in Example 6.9 below).

If $n=1, g$ is the identity map, then, $\operatorname{dim} \mathscr{E}(g)=\mathrm{m}(g)=1$, and Lemma 6.7 holds trivially. Let us now assume that $n \geq 2$. Denote by $F$ an invariant critical class of $g$, as defined in Lemma 4.7, let $N=\{1, \ldots, n\} \backslash F$, and put $h=\widetilde{g_{N N}}$, where $g_{N N}=\mathrm{r}_{N} \circ g \circ \mathrm{i}_{N}$, with the notation of Section 4. Since $g \geq I$, we have $g_{N N} \geq I$, so $h \geq I$.

Corollary 6.2 allows us to write for each $1 \leq i \leq n, g_{i}(x)=\max _{p \in \mathscr{P}_{i}} p \cdot x$, where $\mathscr{P}_{i} \subset \partial g_{i}(0)$ is a finite set of stochastic vectors. Consider now, for all $i \in N$, the partition $\mathscr{P}_{i}=\mathscr{N}_{i} \cup \mathscr{F}_{i}$ where $\mathscr{N}_{i}=\left\{p \in \mathscr{P}_{i} \mid p_{j}=0, \forall j \in F\right\}$, and $\mathscr{F}_{i}=\mathscr{P}_{i} \backslash \mathscr{N}_{i}$. We have $h_{i}(y)=\max _{p \in \mathscr{N}_{i}} p \cdot \mathrm{i}_{N}(y)$ for all $i \in N, y \in \mathbb{R}^{N}$ (in particular, $\mathscr{N}_{i} \neq \varnothing$, for all $i \in N$, because $h \geq I$ takes finite values), and, for all $x \in \mathbb{R}^{n}$,

$$
\begin{aligned}
& g_{i}(x)=h_{i} \circ \mathrm{r}_{N}(x) \vee \max _{p \in \mathscr{F}_{i}} p \cdot x \quad \forall i \in N, \\
& g_{i}(x)=\left(g_{F F} \circ \mathrm{r}_{F}(x)\right)_{i} \quad \forall i \in F .
\end{aligned}
$$

From (17), we get the disjoint union $\mathscr{G}^{\mathrm{c}}(g)=\mathscr{G}^{\mathrm{c}}(h) \cup \mathscr{G}^{\mathrm{c}}\left(g_{F F}\right)$, so $\mathrm{m}(g)=\mathrm{m}(h)+1$. By the induction assumption, $\mathscr{E}(h)$ is a convex set of dimension $\mathrm{m}(h)$, which implies that we can find a bounded convex set $U \subset \mathscr{E}(h)$ of dimension $\mathrm{m}(h)$. We shall complete the elements of $U$ to get eigenvectors of $g$ as follows. To each $u \in U$, and $\lambda \in \mathbb{R}$, associate the vector $v(\lambda, u) \in \mathbb{R}^{n}$ such that $\mathbf{r}_{N}(v(\lambda, u))=u$, and $\mathbf{r}_{F}(v(\lambda, u))=\lambda$ (the constant vector). We get from (25b) and Lemma 4.9,

$$
g_{i}(v(u, \lambda))=\left(g_{F F}\right)_{i}(\lambda)=\lambda=v(u, \lambda)_{i} \quad \forall i \in F
$$

and we get from (25a),

$$
g_{i}(v(u, \lambda))=h_{i}(u) \vee \max _{p \in \mathscr{F}_{i}} p \cdot v(u, \lambda)=u_{i} \vee \max _{p \in \mathscr{F}_{i}} p \cdot v(u, \lambda) \quad \forall i \in N .
$$

Since for all $i \in N$ and $p \in \mathscr{F}_{i}, p_{j} \neq 0$ for some $j \in F$, we get $p \cdot v(u, \lambda)=\sum_{j \in N} p_{j} u_{j}+$ $\sum_{j \in F} p_{j} \lambda \rightarrow-\infty$ when $\lambda \rightarrow-\infty$. Since $U$ is bounded and the sets $\mathscr{F}_{i}$ are finite, the limit is uniform in $u \in U$ and $p \in \mathscr{F}_{i}$. Therefore, we get from (27) that

$$
g_{i}(v(u, \lambda))=u_{i}=(v(u, \lambda))_{i} \quad \forall i \in N,
$$

holds for all $u \in U$ and $\lambda \leq \lambda_{0}$, for some $\lambda_{0} \in \mathbb{R}$. Combining (26) with (28), we get that $g(v(u, \lambda))=v(u, \lambda)$ for all $u \in U$ and $\lambda \leq \lambda_{0}$, which shows that $\mathscr{E}(g)$ has dimension $\mathrm{m}(h)+1=$ $\mathbf{m}(g)$. 
We obtain in passing some information on the restriction of $\mathscr{E}(g)$ to the set of invariant critical classes. If $I \subset\{1, \ldots, n\}$, we denote by $1_{I} \in \mathbb{R}^{n}$ the vector such that $\left(1_{I}\right)_{i}=1$ if $i \in I$, and $\left(1_{I}\right)_{i}=0$ otherwise.

Corollary 6.8. Let $g$ denote a convex piecewise affine monotone bihomogeneous map with invariant critical classes $F_{1}, \ldots, F_{r}$. Then, the restriction of $\mathscr{E}(g)$ to $F_{1} \cup \cdots \cup F_{r}$ is equal to $\mathbb{R} 1_{F_{1}}+\cdots+\mathbb{R} 1_{F_{r}}$.

Proof. The reduction in the proof of Theorem 6.6 shows that we can assume that $g \geq I$, so that we are in the situation of Lemma 6.7. The corollary is obtained by a straightforward variant of the induction argument of Lemma 6.7, in which $F$ is replaced by $F_{1} \cup \cdots \cup F_{r}$, and $v(u, \lambda)$ is replaced by the vector $v\left(u, \lambda_{1}, \ldots, \lambda_{r}\right)$ defined by $\mathbf{r}_{N}\left(v\left(u, \lambda_{1}, \ldots, \lambda_{r}\right)\right)=u$ and $\mathbf{r}_{F_{i}}\left(v\left(u, \lambda_{1}, \ldots, \lambda_{r}\right)\right)=\lambda_{i}$ (the constant vector), with $\lambda_{1}, \ldots, \lambda_{r} \in \mathbb{R}$, and $u \in U$. The proof of Lemma 6.7 shows that for $\max _{i} \lambda_{i}$ close enough to $-\infty, v\left(u, \lambda_{1}, \ldots, \lambda_{r}\right)$ is an eigenvector of $g$. Then, for all $\lambda \in \mathbb{R}$, $v\left(\lambda+u, \lambda+\lambda_{1}, \ldots, \lambda+\lambda_{r}\right)$ is an eigenvector of $g$, and since $\left(\lambda+\lambda_{1}, \ldots, \lambda+\lambda_{r}\right)$ can take any value in $\mathbb{R}^{r}$, this implies that the restriction of $\mathscr{E}(g)$ to $F_{1} \cup \cdots \cup F_{r}$ contains $\mathbb{R}_{F_{1}}+\cdots+\mathbb{R} 1_{F_{r}}$. The other inclusion follows from the first assertion of Theorem 3.4.

\subsection{Examples}

Example 6.9. Let $a_{1}, a_{2}, a_{3}>0$, and consider

$$
f: \mathbb{R}^{3} \rightarrow \mathbb{R}^{3}, \quad f(x)=\left(\begin{array}{l}
x_{1} \vee\left(-a_{1}+\left(x_{2}+x_{3}\right) / 2\right) \\
x_{2} \vee\left(-a_{2}+\left(x_{1}+x_{3}\right) / 2\right) \\
x_{3} \vee\left(-a_{3}+\left(x_{1}+x_{2}\right) / 2\right)
\end{array}\right)
$$

We have $f(0)=0$ and $f_{0}^{\prime}(x)=x$. Therefore, Theorem 6.6 states that $\mathscr{E}(f)$ has dimension 3 . Indeed, it is immediate to check that $\mathscr{E}(f)=\left\{x \mid x_{i} \geq-a_{i}+\left(x_{k}+x_{j}\right) / 2, \forall 1 \leq i \leq 3, k \neq i, j \neq i\right\}$, and this set has dimension 3 . Since $\mathscr{E}(f)$ is invariant by the translations of vector $(\lambda, \lambda, \lambda)$, for all $\lambda \in \mathbb{R}$, it is convenient to represent the projection of $\mathscr{E}(f)$ on any plane orthogonal to the direction $(1,1,1)$, which is as follows (we take $a_{1}=1 / 2, a_{2}=1, a_{3}=2$, the point $(0,0,0)$ is represented by a bold point, the projection of the eigenspace is the shaded region).

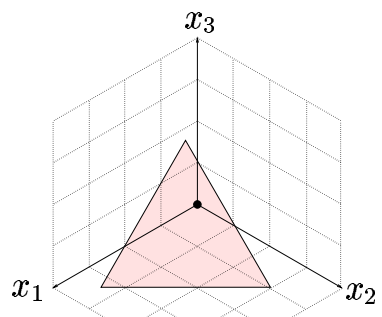

By deforming this picture, it should be obvious that in the limit case $a_{1}=a_{2}=0, a_{3}>0$, the eigenspace $\mathscr{E}(f)$, which looks as follows, 


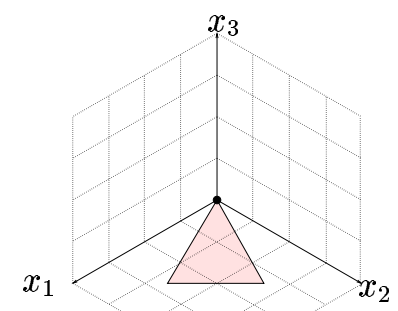

has still dimension 3 . Let us check this. We have in this case

$$
f_{0}^{\prime}(x)=\left(\begin{array}{c}
x_{1} \vee\left(x_{2}+x_{3}\right) / 2 \\
x_{2} \vee\left(x_{1}+x_{3}\right) / 2 \\
x_{3}
\end{array}\right) .
$$

We see that $f_{0}^{\prime}$ has the three critical classes $\{1\},\{2\},\{3\}$, and, by Theorem $6.6, \mathscr{E}(f)$ has dimension 3 . It is instructive to illustrate the proof of Lemma 6.7 by the example of $g=f_{0}^{\prime}$. There is only one invariant critical class, $F=\{3\}$. We have $N=\{1,2\}$, and $h(y)=y$, for all $y \in \mathbb{R}^{2}$. The representation (29) corresponds to $\mathscr{N}_{1}=\{(1,0,0)\}, \mathscr{F}_{1}=(0,1 / 2,1 / 2), \mathscr{N}_{2}=\{(0,1,0)\}$, $\mathscr{F}_{2}=(1 / 2,0,1 / 2)$. Any $u=\left(u_{1}, u_{2}\right) \in \mathbb{R}^{2}$ is an eigenvector of the identity map $h$. The last step of the proof of the lemma shows that for $\lambda$ negative enough, $v(u, \lambda)=\left(u_{1}, u_{2}, \lambda\right)$ is an eigenvector of $f_{0}^{\prime}$. More precisely, it is not difficult to see that the eigenspace of $f_{0}^{\prime}$ looks as follows

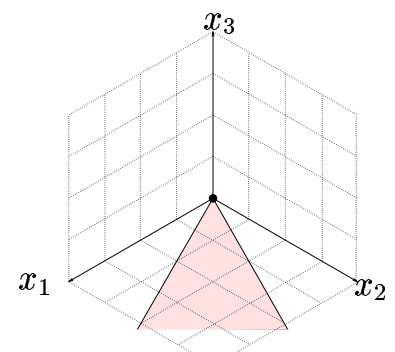

where the shaded region now extends infinitely in the direction $x_{3} \rightarrow-\infty$. (In this example, $\mathscr{E}\left(f_{0}^{\prime}\right)$ is the contingent cone to $\mathscr{E}(f)$ at the point 0 .)

Example 6.10. We next present a variant of Example 6.9, for which $f$ is of cyclicity 2. Let $a_{1}, a_{2}, a_{3}>0$, and

$$
f: \mathbb{R}^{3} \rightarrow \mathbb{R}^{3}, \quad f(x)=\left(\begin{array}{l}
x_{2} \vee\left(-a_{1}+\left(x_{1}+x_{3}\right) / 2\right) \\
x_{1} \vee\left(-a_{2}+\left(x_{2}+x_{3}\right) / 2\right) \\
x_{3} \vee\left(-a_{3}+\left(x_{1}+x_{2}\right) / 2\right)
\end{array}\right) .
$$


We have $f(0)=0$, and $\partial f(0)=\left\{f^{\prime}(0)\right\}$ with

$$
f^{\prime}(0)=\left(\begin{array}{lll}
0 & 1 & 0 \\
1 & 0 & 0 \\
0 & 0 & 1
\end{array}\right),
$$

which gives the critical graph:

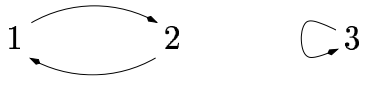

Therefore, $\mathscr{G}^{\mathrm{c}}(f)$ is the union of two strongly connected components, $\mathscr{G}_{1}$, and $\mathscr{G}_{2}$, with respective sets of nodes $\{1,2\}$ and $\{3\}$ and cyclicities 2 and 1 , which implies that $f$ has cyclicity $\mathrm{c}(f)=$ $\operatorname{lcm}(2,1)=2$. An immediate computation shows that

$$
\mathscr{E}(f)=\left\{x \in \mathbb{R}^{3} \mid a_{3}+x_{3} \geq x_{1}=x_{2} \geq-2\left(a_{1} \wedge a_{2}\right)+x_{3}\right\} .
$$

This two dimensional convex set is represented by the vertical bold segment on the following figure:

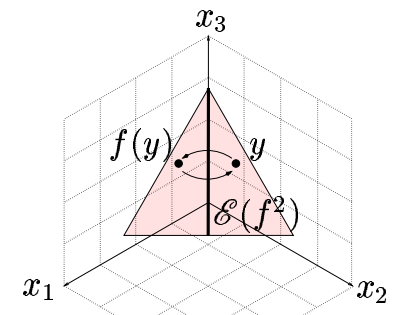

Corollary 5.8 says that the union of periodic orbits of $f$ is equal to $\mathscr{E}\left(f^{2}\right)$. A new computation shows that $\mathscr{E}\left(f^{2}\right)$ is equal to the triangular region determined by the inequalities

$$
\begin{aligned}
& x_{1} \geq-\left(a_{1} \wedge a_{2}\right)+\left(x_{2}+x_{3}\right) / 2, \\
& x_{2} \geq-\left(a_{1} \wedge a_{2}\right)+\left(x_{1}+x_{3}\right) / 2, \\
& x_{3} \geq-a_{3}+\left(x_{1}+x_{2}\right) / 2,
\end{aligned}
$$

which is depicted in the figure. On this example, Theorem 6.6 predicts that $\mathscr{E}(f)$ is of dimension 2, and Proposition 5.3, combined with Theorem 6.6, predicts that $\mathscr{E}\left(f^{2}\right)$ is of dimension c $\left(\mathscr{G}_{1}\right)+$ $\mathrm{c}\left(\mathscr{G}_{2}\right)=3$. It is easy to see that $f$ acts on $\mathscr{E}\left(f^{2}\right)$ as the reflection with respect to the plane $x_{1}=x_{2}$. As an illustration, a periodic orbit $\{y, f(y)\}$ is depicted on the figure.

Example 6.11. Consider any convex monotone homogeneous map $f: \mathbb{R}^{n} \rightarrow \mathbb{R}^{n}$, whose eigenspace has dimension $n$. We show here how, in that case, Theorem 6.6 allows us to characterize the set $\mathscr{E}(f)$. We assume without loss of generality, that the eigenvalue is 0 . Since the dimension of $\mathscr{E}(f)$ is $n$, the number of critical classes must be equal to the dimension, $n$, hence $f \geq I$, or equivalently, $f=I \vee g$, for some convex monotone homogeneous map $g$. Then,

$$
\mathscr{E}(f)=\left\{x \in \mathbb{R}^{n} \mid x \geq P x-g^{*}(p) \forall P \in \operatorname{dom} g^{*}\right\} .
$$

$\mathrm{RR} n^{\circ} 4273$ 
Half-spaces of the form $x_{i} \geq P_{i} x-g_{i}^{*}(p)$ involved in this definition are special. Indeed, the later inequality can be rewritten as

$$
x_{i} \geq \sum_{j \neq i} \alpha_{j} x_{j}+\gamma
$$

with $0 \leq \alpha_{j}, \gamma \in \mathbb{R}$, and $\sum_{j \neq i} \alpha_{j}=1$. Using (30) and (31), we get that a subset of $\mathbb{R}^{n}$ of nonempty interior is the eigenspace of a convex monotone homogeneous map $f: \mathbb{R}^{n} \rightarrow \mathbb{R}^{n}$ if, and only if, it is the intersection of half-spaces of the form (31). This implies, for instance, that the region at the left hand side of the following figure is the eigenspace of a convex monotone homogeneous map:

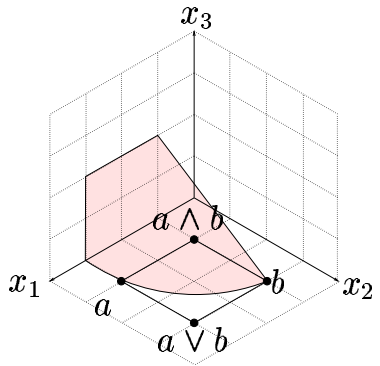

$f$ convex

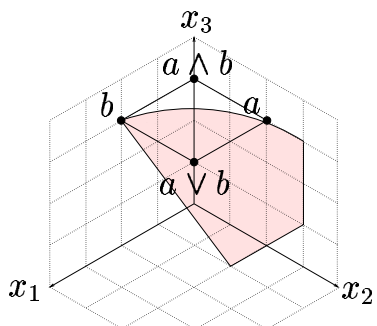

$f$ concave

The fact that $\mathscr{E}(f)$ is an inf-subsemilattice of $\mathbb{R}^{n}$ (but not a sup-subsemilattice) is illustrated by the four points $a, b, a \vee b, a \wedge b$. Eigenspaces of concave monotone homogeneous maps have symmetric shapes, like the one at the right hand side of the figure.

Example 6.12. The following example shows that for a non-convex piecewise affine monotone homogeneous map $f$, the dimension of the eigenspace is ill-defined. Consider $f: \mathbb{R}^{3} \rightarrow \mathbb{R}^{3}$,

$$
f(x)=\left(\begin{array}{rl}
\left(x_{1} \wedge x_{2} \wedge x_{3}\right) & \vee\left(x_{1} \wedge\left(-1+x_{2}\right) \wedge\left(1+x_{3}\right)\right) \\
\left(2+x_{1}\right) \wedge & \wedge x_{2} \wedge\left(3+x_{3}\right) \\
\left(x_{1} \wedge x_{2} \wedge x_{3}\right)
\end{array}\right) .
$$

Then, it is easy to check that $f=f^{2}$, and that the eigenspace of $f$ is the following flag shaped set:

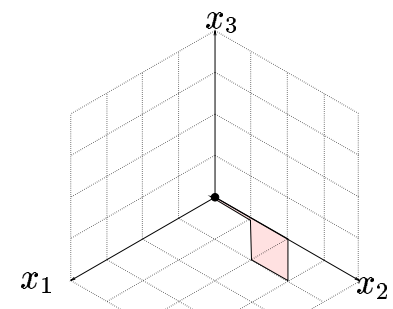

Explicitly, $\mathscr{E}(f)=K_{1} \cup K_{2}$, where $K_{1}=\{(\lambda, \lambda+t, \lambda) \mid \lambda \in \mathbb{R}, 0 \leq t \leq 1\}$, and $K_{2}=$ $\{(\lambda, \lambda+t, \lambda+s) \mid \lambda \in \mathbb{R}, 1 \leq t \leq 2,-1 \leq s \leq 0\}$, which shows that the "local dimension" 
of the eigenspace near a point $x \in \mathscr{E}(f)$ is 2 (if $x$ is in the relative interior of $K_{1}$ ) or 3 (if $x$ is in the interior of $K_{2}$ ). Although we do not need this here, let us mention that there is a systematic technology to build such examples, which originates from max-plus algebra: $f$ is a projector on the max-plus semimodule generated by the columns of the matrix

$$
\left(\begin{array}{ccc}
0 & 0 & 0 \\
0 & 1 & 2 \\
0 & -1 & -1
\end{array}\right) .
$$

See [CGQ97] for details.

\subsection{Computing $\mathscr{G}^{\mathrm{c}}(f)$}

To conclude this section, we give a polynomial time algorithm to compute

$$
\mathscr{G}^{\mathrm{f}}\left(\operatorname{co}\left(\mathscr{Q}_{1} \times \cdots \times \mathscr{Q}_{n}\right)\right)
$$

given finite sets of stochastic vectors $\mathscr{Q}_{1}, \ldots, \mathscr{Q}_{n}$. This algorithm allows us, in particular, to compute the critical graph of a piecewise affine convex monotone homogeneous map $f$, provided that an eigenvector $u \in \mathbb{R}^{n}$ of $f$ is known. Indeed, the coordinates of $f$ are of the form

$$
f_{i}(x)=\max _{p \in \mathscr{P}_{i}}\left(p \cdot x-f_{i}^{*}(p)\right)
$$

where the $\mathscr{P}_{i}$ are finite sets of stochastic vectors, and, setting $\mathscr{Q}_{i}=\left\{p \in \mathscr{P}_{i} \mid p \cdot u-f_{i}^{*}(p)=\right.$ $\left.f_{i}(u)\right\}$, it follows from [RW98, Th. 10.31] that $\partial f_{i}(u)=\operatorname{co} \mathscr{Q}_{i}$, hence $\partial f(u)=\operatorname{co}\left(\mathscr{Q}_{1} \times \cdots \times \mathscr{Q}_{n}\right)$. Since $\mathscr{G}^{\mathrm{c}}(f)=\mathscr{G}^{\mathrm{f}}(\partial f(u))$, the problem of computing $\mathscr{G}^{\mathrm{c}}(f)$ reduces to that of computing (33).

To write the algorithm, it will be convenient to consider more generally a finite family $\left\{\mathscr{Q}_{i}\right\}_{i \in I}$, where $\mathscr{Q}_{i} \subset \mathbb{R}^{I}$ is a finite set of substochastic vectors. For any rectangular set $\mathscr{Q}$ of substochastic matrices, we define $\mathscr{G}^{\mathrm{f}}(\mathscr{Q})$ as the union of the graphs of the matrices $P_{F F}$, where $P \in \mathscr{Q}, F$ is a final class of $P$, and $P_{F F}$ is a stochastic matrix (this definition is consistent with the one of $\mathscr{G}^{\mathrm{c}}(f)$ for a monotone subhomogeneous map $f$, that we gave in $\S 1.4$ ).

The algorithm can be specified as a recursive function, with input $\left\{\mathscr{Q}_{i}\right\}_{i \in I}$, and output $\mathscr{G}^{\mathrm{f}}\left(\operatorname{co} \times_{i \in I} \mathscr{Q}_{i}\right)$. The function first builds, for all $i \in I$, the subset $\mathscr{Q}_{i}^{\prime} \subset \mathscr{Q}_{i}$ of row vectors with row sum 1, together with the graph $G=\mathscr{G}\left(\times_{i \in I} \mathscr{Q}_{i}^{\prime}\right)=\cup_{P \in \times_{i \in I} \mathscr{Q}_{i}^{\prime}} \mathscr{G}(P)$. If all the strongly connected components of $G$ are trivial (we say that a strongly connected component is trivial if it has only one node and no arcs), the function returns the empty graph (with no nodes). Otherwise, we proceed as follows. We denote by $F$ the union of final classes of $G$, and put $N=I \backslash F$. (The set $F$ is indeed the union of invariant final classes of $f$ when $\operatorname{co} \times_{i \in I} \mathscr{Q}_{i}=\partial f(u)$, see Lemma 4.7.) For $i \in N$, we define the sets $\mathscr{Q}_{i}^{\prime \prime} \subset \mathbb{R}^{N}$ of row vectors obtained by restricting to $N$ the vectors $p \in \mathscr{Q}_{i}^{\prime}$ such that $p_{j}=0$, for all $j \in F$. We denote by $\left.G\right|_{F}$ the restriction of $G$ to $F$. The identity

$$
\mathscr{G}^{\mathrm{f}}\left(\operatorname{co} \times_{i \in I} \mathscr{Q}_{i}\right)=\left.G\right|_{F} \cup \mathscr{G}^{\mathrm{f}}\left(\operatorname{co} \times_{i \in N} \mathscr{Q}_{i}^{\prime \prime}\right),
$$

yields a recursive algorithm to compute $\mathscr{G}^{\mathrm{f}}\left(\operatorname{co} \times_{i \in I} \mathscr{Q}_{i}\right)$. (The identity (35) is similar to (17).) 
Applying this algorithm to the case of Example 2.4, we get $\mathscr{Q}_{1}=$ $\{(1,0,0),(1 / 2,1 / 2,0),(1 / 2,0,1 / 2)\}, \mathscr{Q}_{2}=\{(0,1,0),(2 / 3,1 / 3,0)\}, \mathscr{Q}_{3}=\{(0,0,1)\}$, $\mathscr{Q}_{i}^{\prime}=\mathscr{Q}_{i}, F=\{3\}, \mathscr{Q}_{1}^{\prime \prime}=\{(1,0),(1 / 2,1 / 2)\}, \mathscr{Q}_{2}^{\prime \prime}=\{(0,1),(2 / 3,1 / 3)\}$, so that (35) shows that $\mathscr{G}^{\mathrm{f}}\left(\operatorname{co}\left(\mathscr{Q}_{1} \times \mathscr{Q}_{2} \times \mathscr{Q}_{3}\right)\right)$ is the union of a loop at the node 3 , and of the complete graph with nodes 1,2 .

\section{Stochastic Control Interpretation}

In this section, we briefly explain how the above results can be applied to stochastic control. This application also makes the results more intuitive. See for instance [Whi86], or [HLL96] for more background on stochastic control.

A Markov control model with state space $\{1, \ldots, n\}$ is a 4-uple ( $A, \quad\left\{A_{i}\right\}_{1 \leq i \leq n}, \quad\left\{r_{i}\right\}_{1 \leq i \leq n}, \quad\left\{P_{i}\right\}_{1 \leq i \leq n}$ ), where: $A$ is a set, called action space; for each state $1 \leq i \leq n, A_{i}$ is a nonempty subset of $A$, whose elements are interpreted as possible actions; $r_{i}$ is a map from $A_{i}$ to $\mathbb{R}$, the image $r_{i}^{a}$ of $a$ is interpreted as an instantaneous reward received when action $a$ is performed in state $i ; P_{i}$ is a map from $A_{i}$ to the set $\mathscr{S}_{n}$ of stochastic vectors, and the $j$-th entry, $P_{i j}^{a}$, of the image $P_{i}^{a}$ of $a$ is interpreted as the transition probability from state $i$ to state $j$, when the action $a$ is performed. It will be convenient to assume that $A$ is a topological space equipped with its Borel $\sigma$-algebra, that the $A_{i}$ are Borel sets, that the maps $r_{i}$ and $P_{i}$ are measurable, and that

$$
\text { for all } x \in \mathbb{R}^{n}, \quad \sup _{a \in A_{i}}\left(r_{i}^{a}+P_{i}^{a} x\right) \quad \text { is attained (and finite) }
$$

(this is the case, in particular, if $A_{i}$ is compact, $r_{i}$ is upper semi-continuous, and $P_{i}$ is continuous).

The intuitive notion of strategy, i.e. of causal rule telling which action to choose, is captured by the following definitions. An history is a sequence of states $\left(i_{0}, i_{1}, \ldots\right)$, a partial history is a finite sequence $\left(i_{0}, \ldots, i_{k}\right)$. A (randomized) strategy is a sequence $\gamma=\left(\gamma_{0}, \gamma_{1}, \ldots\right)$ where $\gamma_{k}$ is a map which to a partial history $\left(i_{0}, \ldots, i_{k}\right)$ associates a probability measure $\gamma_{k}^{i_{0}, \ldots, i_{k}}$ on $A$ such that $A_{i_{k}}$ has probability 1 , i.e. $\gamma_{k}^{i_{0}, \ldots, i_{k}}\left[A_{i_{k}}\right]=1$ : the action at time $k, a_{k} \in A_{i_{k}}$, will be chosen with probability $\gamma_{k}^{i_{0}, \ldots, i_{k}}$. We say that $\gamma$ is deterministic if $\gamma_{k}^{i_{0}, \ldots, i_{k}}$ is a Dirac measure. We say that $\gamma$ is Markovian if $\gamma_{k}^{i_{0}, \ldots, i_{k}}$ only depends of $k$ and $i_{k}$, and that a Markovian $\gamma$ is stationary if $\gamma_{k}$ is independent of $k$. Markovian stationary policies are obtained by choosing, for all $1 \leq i \leq n$, a probability measure $\tau_{i}$ on $A$ such that $\tau_{i}\left[A_{i}\right]=1$, and taking $\gamma_{k}^{i_{0}, \ldots, i_{k}}=\tau_{i_{k}}$. We denote by $\tau^{\infty}$ the (Markovian, stationary) strategy $\gamma$ built in this way. For any strategy $\gamma$, and initial state $i_{0}$, we consider a (state, action) stochastic process $\left(\xi_{0}, \alpha_{0}\right),\left(\xi_{1}, \alpha_{1}\right), \ldots$ with values in $\{1, \ldots, n\} \times A$, such that $\xi_{0}=i_{0}$, the law of $\alpha_{k}$ knowing $\xi_{0}, \ldots, \xi_{k}, \alpha_{0} \in A_{\xi_{0}}, \ldots, \alpha_{k-1} \in A_{\xi_{k-1}}$ is equal to $\gamma_{k}^{\xi_{0}, \ldots, \xi_{k}}$, and the law of $\xi_{k}$ knowing $\xi_{0}, \ldots, \xi_{k-1}, \alpha_{0} \in A_{\xi_{0}}, \ldots, \alpha_{k-1} \in A_{\xi_{k-1}}$ is equal to $P_{\xi_{k-1} \xi_{k}}^{\alpha_{k-1}}$. When $\gamma$ is Markovian and stationary, $\xi_{k}$ simply becomes a time homogeneous Markov chain with initial state $i$ and transition matrix $P^{\tau}$,

$$
P_{i j}^{\tau}=\int_{A} P_{i j}^{a} d \tau_{i}(a)
$$


The ergodic control problem consists in finding a strategy $\gamma$ which maximizes, for all initial states $1 \leq i \leq n$, the mean reward per time unit:

$$
\mu_{i}^{\gamma}=\liminf _{N \rightarrow \infty} \frac{1}{N} \mathbb{E}^{\gamma, i}\left(r_{\xi_{0}}^{\alpha_{0}}+\cdots+r_{\xi_{N-1}}^{\alpha_{N-1}}\right)
$$

We denote by

$$
\mu_{i}=\sup _{\gamma} \mu_{i}^{\gamma}
$$

the optimum mean reward.

A closely related problem is to solve, for large $N$, the horizon $N$ problem, which consists in maximizing

$$
v_{i}^{\gamma}(N)=\mathbb{E}^{\gamma, i}\left(r_{\xi_{0}}^{\alpha_{0}}+\cdots+r_{\xi_{N-1}}^{\alpha_{N-1}}+\phi_{\xi_{N}}\right)
$$

where the final reward $\phi$ is a map from $\{1, \ldots, n\}$ to $\mathbb{R}$, or equivalently an element of $\mathbb{R}^{n}$. We set

$$
v_{i}(N)=\sup _{\gamma} v_{i}^{\gamma}(N)
$$

The vector $v(N) \in \mathbb{R}^{n}$ is called the value function. When $\gamma=\tau^{\infty}$, we shall simply write $\mu^{\tau}$ and $v^{\tau}$, instead of $\mu^{\tau^{\infty}}$ and $v^{\tau^{\infty}}$, respectively.

The study of both the ergodic control and finite horizon problems relies on the dynamic programming operator, or Hamiltonian, $f: \mathbb{R}^{n} \rightarrow \mathbb{R}^{n}$,

$$
f_{i}(x)=\sup _{a \in A_{i}}\left(r_{i}^{a}+P_{i}^{a} x\right)
$$

It is obvious from (40) (and well known, see e.g. [Whi86, Chap. 22, Th. 6.1]), that the map $f$ is monotone, (additively) homogeneous, and convex. Conversely, the Legendre-Fenchel duality theorem shows that a convex monotone homogeneous map $f$ can be written as

$$
f_{i}(x)=\sup _{p \in \operatorname{dom} f_{i}^{*}}\left(p \cdot x-f_{i}^{*}(p)\right)
$$

Since $\operatorname{dom} f_{i}^{*}$ is included in the set of stochastic vectors (Proposition 2.1), and since $p \mapsto f_{i}^{*}(p)$ is lower semi-continuous, this is clearly of the form (40) (although dom $f_{i}^{*}$ need not be compact, note that property (36) is satisfied). Moreover, when the action space $A$ is finite, $\operatorname{dom} f_{i}^{*}=\operatorname{co} A_{i}$ is a polyhedron, and, by Proposition 6.1, $f_{i}$ is piecewise affine. Therefore:

Proposition 7.1. The dynamic programming operators of Markov control models with state space $\{1, \ldots, n\}$ are exactly the convex, monotone homogeneous maps $\mathbb{R}^{n} \rightarrow \mathbb{R}^{n}$. Moreover, Markov control models with finite action spaces correspond to piecewise affine maps.

(The representation (41) provides of canonical form for a Markov control models, in which one can choose, when in state $i$, the transition probability from state $i, p \in \operatorname{dom} f_{i}^{*}$.) 
The value function $v(N)$ can be computed recursively via the dynamic programming equation

$$
v(0)=\phi, \quad v(N)=f(v(N-1))
$$

(see e.g. [HLL96, Th. 3.2.1]). Therefore, an eigenvector $u$, with associated eigenvalue $\lambda$, yields a stationary solution of the dynamic programming equation (42), $v(N)=N \lambda+u$, corresponding to the final reward $\phi=u$. Such stationary solutions are of economic interest. Indeed, when $f(u)=$ $\lambda+u$, we set

$$
\bar{A}_{i}=\left\{a \in A_{i} \mid \lambda+u_{i}=r_{i}^{a}+P_{i}^{a} u\right\}
$$

$\left(\bar{A}_{i} \neq \emptyset\right.$ thanks to (36)) and build a Markovian stationary strategy $\tau^{\infty}$ by picking any probability measure $\tau$ such that $\tau\left[\bar{A}_{i}\right]=1$. A standard result, that we shall not prove (results of this kind can be found in [Whi86, Chap. 31] or [HLL96, Th. 5.2.4]), states that such a strategy is optimal both for the ergodic control problem and for all the finite horizon problems with final reward $\phi=$ $u$, which means, loosely speaking, that taking $\phi=u$ makes it possible for the player to behave (optimally) in the short term as he would in the long term (see [YK92] for more details on the economic interpretation).

Another motivation for describing the set of eigenvectors comes from (42): Corollary 5.7 shows that

$$
v(N c(f))-N c(f) \lambda \text { converges, when } N \rightarrow \infty,
$$

and the possible values of the limit are precisely the eigenvectors of $f^{c(f)}$.

Critical classes also have a stochastic control interpretation. If $\gamma=\tau^{\infty}$ is a Markovian stationary strategy, we define the vector $r^{\tau} \in(\mathbb{R} \cup\{-\infty\})^{n}$ by

$$
r_{i}^{\tau}=\int_{A} r_{i}^{a} d \tau_{i}(a)
$$

If $F$ is a final class of $P^{\tau}$, we denote by $m_{F}^{\tau}$ the unique invariant measure of the $F \times F$ submatrix of $P^{\tau}$, and by $r_{F}^{\tau} \in \mathbb{R}^{F}$ the restriction of the vector $r^{\tau}$ to $F$.

Proposition 7.2. Let us assume that the dynamic programming operator $f$ has an eigenvector $u$, with associated eigenvalue $\lambda$. Then,

$$
\lambda=\max _{1 \leq i \leq n} \mu_{i}^{\gamma}=\max _{\substack{\tau \infty \\ 1 \leq i \leq n}} \mu_{i}^{\tau} \quad \text { and } \max _{1 \leq i \leq n} \mu_{i}^{\tau}=\max _{F \text { fin nal class of } P^{\tau}} m_{F}^{\tau} r_{F}^{\tau},
$$

where the first two max are taken over randomized strategies $\gamma$, and randomized Markovian stationary strategies $\tau^{\infty}$, respectively. Moreover, if all the $A_{i}$ are compact, and all the maps $r_{i}$ and $P_{i}$ are continuous, the elements of $\mathscr{C}^{\mathrm{c}}(f)$ are precisely the $F$ such that $m_{F}^{\tau} r_{F}^{\tau}=\lambda$.

Thus, the critical classes of $f$ are exactly the maximal final classes of randomized Markovian stationary strategies that are optimal for the ergodic control problem. (Considering randomized strategies is essential for this equivalence to hold, even if the maximum in (44) is also attained by deterministic strategies.) In particular, the critical nodes, i.e. the nodes of $\mathscr{G}^{\mathrm{c}}(f)$, are exactly the nodes which are visited infinitely often, almost surely, by the trajectory of at least one optimal Markovian stationary policy. 
Proof. The equalities in (44) are standard results of stochastic control (see for instance [HLL96, Th. 5.2.4] for the first two equalities; the last equality follows from the ergodic theorem for reducible Markov chains, see for instance [Whi86, Ch. 31,§6]). We will only prove here the characterization of critical classes.

If $F \in \mathscr{C}^{\mathrm{c}}(f), F$ is a final class of a stochastic matrix $Q \in \partial f(u)$, for some eigenvector $u$ of $f$. Subdifferentiating (40) at $x=u$, we get, thanks to the technical assumption on $A_{i}, r_{i}$ and $P_{i}$,

$$
\partial f_{i}(u)=\operatorname{co}\left\{P_{i}^{a} \mid a \in \bar{A}_{i}\right\}
$$

see [RW98, Th. 10.31]. Thus, we can write $Q_{i}$ as a convex combination, $Q_{i}=\sum_{a \in \bar{A}_{i}} \alpha_{i}^{a} P_{i}^{a}$, where the $\alpha_{i}^{a}$ are such that $\alpha_{i}^{a} \geq 0, \sum_{a \in \bar{A}_{i}} \alpha_{i}^{a}=1$, and all but finitely many $\alpha_{i}^{a}$ are zero. Consider now the measure $\tau_{i}=\sum_{a \in \bar{A}_{i}} \alpha_{i}^{a} \delta_{a}$ (the associated randomized Markov stationary strategy $\tau^{\infty}$ consists in playing the action $a$ with probability $\alpha_{i}^{a}$, when in state $i$, here $\delta_{a}$ denotes the Dirac probability measure at $a$ ). Averaging the equalities $\lambda+u_{i}=r_{i}^{a}+P_{i}^{a} u$ which hold for all $a \in \bar{A}_{i}$, we get

$$
\lambda+u=r^{\tau}+P^{\tau} u
$$

Since $F$ is a final class of the matrix $P^{\tau}$, restricting (45) to $F$ and left multiplying it by $m_{F}^{\tau}$, we get $\lambda+m_{F}^{\tau} u_{F}=m_{F}^{\tau} r_{F}^{\tau}+m_{F}^{\tau} P_{F F}^{\tau} u_{F}$, and since $m_{F}^{\tau} P_{F F}^{\tau}=m_{F}^{\tau}$, we get $\lambda=m_{F}^{\tau} r_{F}^{\tau}$.

Conversely, let us assume that $F$ is a final class of a matrix $P^{\tau}$ for some Markovian stationary strategy $\tau^{\infty}$, and that $m_{F}^{\tau} r_{F}^{\tau}=\lambda$. For all $a \in A_{i}$, we set

$$
w_{i}^{a}=\lambda+u_{i}-r_{i}^{a}-P_{i}^{a} u \geq 0,
$$

and define the vector $w=\left(\int_{A} w_{i}^{a} d \tau_{i}(a)\right)_{i \in F}$. We have

$$
w=\lambda+u_{F}-r_{F}^{\tau}-P_{F F}^{\tau} u_{F} .
$$

Left multiplying (46) by $m_{F}^{\tau}$ and using again the fact that $m_{F}^{\tau} P_{F F}^{\tau}=m_{F}^{\tau}$, together with $m_{F}^{\tau} r_{F}^{\tau}=\lambda$, we get $m_{F}^{\tau} w=0$. Since all the entries of $m_{F}^{\tau}$ are positive, and those of $w$ are nonnegative, we must have $w=0$. Hence $f_{i}(u)=r_{i}^{\tau}+P_{i}^{\tau} u$, for all $i \in F$, which implies that $P_{i}^{\tau} \in \partial f_{i}(u)$, for all $i \in F$. Taking any element $Q \in \partial f(u)$ such that $Q_{i}=P_{i}^{\tau}$ for $i \in F$, we obtain that $F$ is a final class of $Q$, hence $F \in \mathscr{C}^{\mathrm{c}}(f)$.

\section{References}

[AB99] C. Aliprantis and K. C. Border. Infinite Dimensional Analysis. A Hitchiker's Guide. Springer, 1999.

[AG01] M. Akian and S. Gaubert. A spectral theorem for convex monotone homogeneous maps. In Proceedings of the Satellite Workshop on Max-Plus Algebras, IFAC SSSC'01, Praha, 2001. Elsevier.

[AK87] M. A. Akcoglu and U. Krengel. Nonlinear models of diffusion on a finite space. Probab. Theory Related Fields, 76(4):411-420, 1987. 
[AQV95] M. Akian, J.-P. Quadrat, and M. Viot. Duality between probability and optimization. 1995. Appears in [Gun98].

[AST01] M. Akian, A. Sulem, and M. Taksar. Dynamic optimisation of long term growth rate for a portfolio with transaction costs and logarithmic utility. Mathematical Finance, 11(2):153-188, 2001.

[Bap98] R. B. Bapat. A max version of the Perron-Frobenius theorem. In Proceedings of the Sixth Conference of the International Linear Algebra Society (Chemnitz, 1996), volume 275/276, pages 3-18, 1998.

[BCOQ92] F. Baccelli, G. Cohen, G. J. Olsder, and J.-P. Quadrat. Synchronization and linearity : an algebra for discrete events systems. John Wiley \& Sons, New-York, 1992.

[Ben88] A. Bensoussan. Perturbation Methods in Optimal Control. Wiley/Gauthier-Villars, 1988.

[Bou95] P. Bougerol. Almost sure stabilizability and Riccati's equation of linear systems with random parameters. SIAM J. Control Optim., 33(3):702-717, 1995.

[BP94] A. Berman and R. Plemmons. Nonnegative matrices in the mathematical sciences. Classics in Applied Mathematics. SIAM, 1994.

[Bru73] R. E. Bruck, Jr. Properties of fixed-point sets of nonexpansive mappings in Banach spaces. Trans. Amer. Math. Soc., 179:251-262, 1973.

[CDQV83] G. Cohen, D. Dubois, J.-P. Quadrat, and M. Viot. Analyse du comportement périodique des systèmes de production par la théorie des dioïdes. Rapport de recherche 191, INRIA, Le Chesnay, France, 1983.

[CG79] R. Cuninghame-Green. Minimax algebra. Springer-Verlag, Berlin, 1979.

[CGQ95a] G. Cohen, S. Gaubert, and J.-P. Quadrat. Algebraic system analysis of timed Petri nets. Aug. 1995. Appears in [Gun98].

[CGQ95b] G. Cohen, S. Gaubert, and J.-P. Quadrat. Asymptotic throughput of continuous timed petri nets. In Proceedings of the 34th Conference on Decision and Control, New Orleans, Dec 1995. IEEE.

[CGQ97] G. Cohen, S. Gaubert, and J.-P. Quadrat. Linear projectors in the max-plus algebra. In Proceedings of the IEEE Mediterranean Conference, Cyprus, 1997. IEEE.

[CT80] M. Crandall and L. Tartar. Some relations between non expansive and order preserving maps. Proceedings of the AMS, 78(3):385-390, 1980.

[Del96] C. Dellacherie. Théorie générale du potentiel, I. Astérisque, 236, 1996. 
[FV97] J. A. Filar and K. Vrieze. Competitive Markov decision processes. Springer-Verlag, 1997.

[GG98] S. Gaubert and J. Gunawardena. A non-linear hierarchy for discrete event dynamical systems. In Proc. of the Fourth Workshop on Discrete Event Systems, (WODES98), Cagliari, Italy, 1998. IEE.

[GG01] S. Gaubert and J. Gunawardena. The Perron-Frobenius theorem for homogeneous, monotone functions. Hewlett-Packard Technical Report 2001-12, HPL-BRIMS, 2001. arXiv:math.FA/0105091.

[GK95] J. Gunawardena and M. Keane. On the existence of cycle times for some nonexpansive maps. BRIMS technical report HPL-BRIMS-95-003, Hewlett-Packard Labs, 1995.

[GM77] M. Gondran and M. Minoux. Valeurs propres et vecteurs propres dans les dioïdes et leur interprétation en théorie des graphes. Bull. Direction Études Recherches Sér. C Math. Informat., 2:i, 25-41, 1977.

[GP97] S. Gaubert and M. Plus. Methods and applications of $(\max ,+)$ linear algebra. In Proceedings of STACS'97, 1997. Rapport de recherche INRIA 3088.

[Gun94] J. Gunawardena. Min-max functions. Discrete Event Dynamic Systems, 4:377-406, 1994.

[Gun98] J. Gunawardena, editor. Idempotency. Publications of the Isaac Newton Institute. Cambridge University Press, 1998.

[Gun01] J. Gunawardena. From max-plus algebra to nonexpansive maps: a nonlinear theory for discrete event systems. To appear in Theoretical Computer Science, 2001.

[HLL96] O. Hernández-Lerma and J.-B. Lasserre. Discrete-Time Markov Control Processes. Springer, 1996.

[KM97] V. N. Kolokoltsov and V. P. Maslov. Idempotent analysis and applications. Kluwer Acad. Publisher, 1997.

[Kol92] V. N. Kolokoltsov. On linear, additive and homogeneous operators in idempotent analysis. 1992. Appears in [MS92].

[KR50] M. G. Krĕ̌n and M. A. Rutman. Linear operators leaving invariant a cone in a Banach space. Amer. Math. Soc. Translation, 1950(26):128, 1950.

[KSK66] J. G. Kemeny, J. Snell, and A. W. Knapp. Denumerable Markov Chains. The University series in higher Mathematics. Van Nostrand, 1966.

[Lan67] E. Lanery. Étude asymptotique des systèmes markoviens à commande. Rev. Française Informat. Recherche Opérationnelle, 1(5):3-56, 1967. 
[Lem01] B. Lemmens. Iteration of nonexpansive maps under the 1-norm. PhD thesis, Vrije Universiteit, Amsterdam, 2001.

[LMS00] G. Litvinov, V. Maslov, and G. Shpiz. Idempotent functional analysis: An algebraical approach. E-print math.FA/0009128, http://arXiv.org, 2000.

[LW94] C. Liverani and M. Wojtkowski. Generalization of the Hilbert metric to the space of positive definite matrices. Pacific. J. Math., 2:339-355, 1994.

[Mas73] V. Maslov. Méthodes Operatorielles. Mir, Moscou, 1973. trad. fr. 1987.

[Mor64] M. Morishima. Equilibrium, stability, and growth: A multi-sectoral analysis. Clarendon Press, Oxford, 1964.

[Mor95] P. D. Moral. Maslov optimization theory: Topological aspects. 1995. Appears in [Gun98].

[MS69] M. Menon and H. Schneider. The spectrum of an operator associated with a matrix. Linear Algebra and its Applications, 2:321-334, 1969.

[MS92] V. P. Maslov and S. N. Samborskiǔ. Idempotent analysis, volume 13 of Advances In Soviet Mathematics. Amer. Math. Soc., Providence, 1992.

[MTRS90] P. D. Moral, T. Thuillet, G. Rigal, and G. Salut. Optimal versus random processes : the nonlinear case. Rapport de recherche, LAAS, 1990.

[NSVL98] R. D. Nussbaum, M. Scheutzow, and S. M. Verduyn Lunel. Periodic points of nonexpansive maps and nonlinear generalizations of the Perron-Frobenius theory. Selecta Math. (N.S.), 4(1):141-181, 1998.

[Nus86] R. D. Nussbaum. Convexity and log-convexity for the spectral radius. Linear Algebra and its Applications, 73:59-122, 1986.

[Nus88] R. D. Nussbaum. Hilbert's projective metric and iterated nonlinear maps. Memoirs of the AMS, 75(391), 1988.

[Nus89] R. D. Nussbaum. Iterated nonlinear maps and Hilbert's projective metric, II. Memoirs of the AMS, 79(401), 1989.

[Nus90] R. D. Nussbaum. Omega limit sets of nonexpansive maps: finiteness and cardinality estimates. Differential Integral Equations, 3(3):523-540, 1990.

[Nus91a] R. D. Nussbaum. Convergence of iterates of a nonlinear operator arising in statistical mechanics. Nonlinearity, 4(4):1223-1240, 1991.

[Nus91b] R. D. Nussbaum. Estimates of the periods of periodic points for nonexpansive operators. Israel J. Math., 76(3):345-380, 1991. 
[NVL99] R. D. Nussbaum and S. M. Verduyn Lunel. Generalizations of the Perron-Frobenius theorem for nonlinear maps. Mem. Amer. Math. Soc., 138(659):viii+98, 1999.

[Osh84] Y. Oshime. Nonlinear Perron-Frobenius problem for weakly contractive transformations. Math. Japon., 29(5):681-704, 1984.

[Puh01] A. Puhalskiı̌. Large Deviations and Idempotent Probability. Number 119 in Monographs and Surveys in Pure and Applied Mathematics. Chapman \& Hall, 2001.

[QP97] J.-P. Quadrat and M. Plus. Min-plus linearity and statistical mechanics. Markov Processes and Related Fields, 3(4):565-587, 1997.

[Qua90] J.-P. Quadrat. Théorèmes asymptotiques en programmation dynamique. C.R.A.S, 311:745-748, 1990.

[Roc70] R. T. Rockafellar. Convex analysis. Princeton University Press Princeton, N.J., 1970.

[Rom67] I. Romanovskiı̌. Optimization of stationary control of discrete deterministic process in dynamic programming. Kibernetika, 3(2):66-78, 1967.

[Rom73] I. V. Romanovsky. On the solvability of Bellman's functional equation for a Markovian decision process. J. Math. Anal. Appl., 42:485-498, 1973. Collection of articles dedicated to Salomon Bochner.

[RS01] D. Rosenberg and S. Sorin. An operator approach to zero-sum repeated games. Israel J. Math., 121:221-246, 2001.

[RW98] R. T. Rockafellar and R. J.-B. Wets. Variational Analysis. Springer, 1998.

[Sab97] C. Sabot. Existence and uniqueness of diffusions on finitely ramified self-similar fractals. Ann. Sci. École Norm. Sup. (4), 30(5):605-673, 1997.

[Sch88] M. Scheutzow. Periods of nonexpansive operators on finite $l_{1}$-spaces. European J. Combin., 9(1):73-81, 1988.

[Sch91] M. Scheutzow. Erratum: "Periods of nonexpansive operators on finite $l_{1}$-spaces". $E u$ ropean J. Combin., 12(2):183, 1991.

[SF77] P. J. Schweitzer and A. Federgruen. The asymptotic behavior of undiscounted value iteration in Markov decision problems. Math. Oper. Res., 2(4):360-381 (1978), 1977.

[SF78] P. J. Schweitzer and A. Federgruen. The functional equations of undiscounted Markov renewal programming. Math. Oper. Res., 3(4):308-321, 1978.

[Sin90] R. Sine. A nonlinear Perron-Frobenius theorem. Proc. Amer. Math. Soc., 109(2):331336, 1990.

[Vin97] J.-M. Vincent. Some ergodic results on stochastic iterative discrete event systems. Discrete Event Dynamic Systems, 7:209-233, 1997. 
[Vor67] N. Vorobyev. Extremal algebra of positive matrices. Elektron. Informationsverarbeitung und Kybernetik, 3, 1967. in Russian.

[We187] D. Weller. Hilbert's metric,part metric and self mappings of a cone. PhD thesis, Universität Bremen, Germany, December 1987.

[Whi86] P. Whittle. Optimization over time. Wiley, 1986. Volume II.

[Woj85] M. Wojtkowski. Invariant families of cones and Lyapunov exponents. Ergod. Th. \& Dynam. Sys., 5:145-161, 1985.

[YK92] S. Y. Yakovenko and L. A. Kontorer. Nonlinear semigroups and infinite horizon optimization. 1992. Appears in [MS92]. 


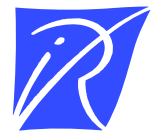

Unité de recherche INRIA Rocquencourt Domaine de Voluceau - Rocquencourt - BP 105 - 78153 Le Chesnay Cedex (France)

Unité de recherche INRIA Lorraine : LORIA, Technopôle de Nancy-Brabois - Campus scientifi que 615, rue du Jardin Botanique - BP 101 - 54602 Villers-lès-Nancy Cedex (France)

Unité de recherche INRIA Rennes : IRISA, Campus universitaire de Beaulieu - 35042 Rennes Cedex (France)

Unité de recherche INRIA Rhône-Alpes : 655, avenue de l'Europe - 38330 Montbonnot-St-Martin (France)

Unité de recherche INRIA Sophia Antipolis : 2004, route des Lucioles - BP 93 - 06902 Sophia Antipolis Cedex (France)

INRIA - Domaine de Voluceau - Rocquencourt, BP 105 - 78153 Le Chesnay Cedex (France)

http://www.inria.fr

ISSN 0249-6399 